\title{
Students' Preferences for Returning to Colleges and Universities During the COVID-19 Pandemic: A
}

Discrete Choice Experiment

Lauren N. Steimle ${ }^{1,}{ }^{*}$, Yuming Sun ${ }^{1}$, Lauren Johnson ${ }^{1}$, Tibor Besedeš ${ }^{2}$, Patricia Mokhtarian ${ }^{3}$, Dima Nazzal ${ }^{1}$

${ }^{1} \mathrm{H}$. Milton Stewart School of Industrial Engineering, Georgia Institute of Technology

${ }^{2}$ School of Economics, Georgia Institute of Technology

${ }^{3}$ School of Civil and Environmental Engineering, Georgia Institute of Technology

*to whom correspondence should be addressed:

755 Ferst Dr NW

Atlanta, GA 30332

Telephone: (404) 894-4659

Email: $\underline{\text { steimle@gatech.edu }}$

ORCIDs of authors:

- Lauren N. Steimle ORCID: 0000-0002-4073-6165

- Yuming Sun ORCID: 0000-0001-8120-1506

- Lauren Johnson ORCID: 0000-0001-8601-390X

- Tibor Besedeš ORCID: 0000-0002-8457-0240

- Patricia Mokhtarian ORCID: 0000-0001-7104-499X

- Dima Nazzal ORCID: 0000-0003-1137-2603 


\section{Abstract}

Importance: Due to the COVID-19 pandemic, institutions of higher education (IHEs) are weighing decisions about when and how to reopen their campuses for the Spring $2021 \mathrm{term}$. Schools are revisiting their plans to use in-person, online, or hybrid (a mixture of in-person and online) modes of course delivery, as well as their safety plans. However, there is still limited knowledge about how to properly plan for campus reopening decisions, including course delivery and campus safety, to maintain enrollment and keep students and faculty safe.

Objectives: To assess 1) students' willingness to comply with health protocols and contrast to their perception of their classmates' compliance, 2) whether students preferred in-person or online learning during a pandemic, and 3) The importance weights of different aspects of campus operations (i.e., modes of course delivery and safety plans) for students when they decide to enroll or defer.

Design, setting, and participants: An internet-based survey of college students took place from June 25, 2020 to July 10, 2020. Participants included 398 industrial engineering students at a medium-size public university in Atlanta, Georgia. The survey included a discrete choice experiment with questions that asked students to choose whether to enroll or defer when presented with hypothetical scenarios related to Fall 2020 modes of course delivery and aspects of campus safety. The survey also asked students about expected compliance with health protocols, whether they preferred in-person or online courses, and sociodemographic information.

Main outcomes and measures: We estimated students' willingness to comply with potential health protocols, choices between in-person and online learning, and the importance of different modes of course delivery and safety measures when deciding to enroll or defer.

Results: The response rate of students who participated in the survey was $20.8 \%$. A latent class model showed three classes of students: those who were "low-concern" (comprising a 29\% expected share of the sample), those who were "moderate-concern" (54\%) and those who were "high-concern" (17\%). We found that scenarios that offered an on-campus experience with large classes delivered online and small classes delivered in-person, strict safety protocols in terms of mask-wearing, testing, and residence halls, and lenient safety protocols in terms of social gatherings were broadly the scenarios with the highest expected enrollment probabilities. The decision to enroll or defer for all students was largely determined by the mode of delivery for courses and the safety measures on campus around COVID-19 testing and mask-wearing. A logistic regression model showed that higher perceived risk of infection of COVID-19, better living suitability for online courses, being older, and less risk seeking were significant factors for a person to choose online learning. Students stated for themselves and their classmates that they would comply with some but not all health protocols against COVID-19, especially those limiting social gatherings.

Conclusions and relevance: The majority of students indicated a preference to enroll during the COVID19 pandemic so long as sufficient safety measures are put in place and all classes were not entirely inperson. As IHEs consider different options for campus operations during pandemics, they should consider the heterogenous preferences among their students. Offering flexibility in course modes may be a way to appeal to many students who vary in terms of their concern about the COVID-19 pandemic. At the same time, since students overall preferred some safety measures placed around mask-wearing and COVID-19 testing on campus, IHEs may want to recommend or require wearing masks and doing 
some surveillance tests for all students, faculty, and staff. Students were expecting themselves and their fellow classmates to comply with some but not all health protocols, which may help IHEs identify

protocols that need more education and awareness, like the limits on social gatherings and the practice of social distancing at social gatherings.

\section{Keywords}

Discrete choice experiment, public health, institution of higher education, college students, latent class model, COVID-19 


\section{Declarations}

Funding: This work was supported by grants from the Thos and Clair Muller Research Endowment Fund of the Georgia Institute of Technology's H. Milton Stewart School of Industrial and Systems Engineering and the Georgia Institute of Technology's Executive Vice President of Research COVID-19 Rapid Response seed grant program.

Conflicts of interest/competing interests:

None

Ethics approval

This study was approved by the Georgia Institute of Technology's Institutional Review Board as an exempt study under Protocol H2O234.

Consent to participate

Participants provided informed consent to participate.

Code availability

Upon request 


\section{Introduction}

During the Spring term of 2020, many institutions of higher education (IHEs) closed their campuses due to the COVID-19 pandemic. Administrators had to grapple with challenging decisions about when and how to reopen their campuses for the Fall 2020 term and, at the time of this writing, are currently revisiting these decisions for the Spring 2021 term. When making these decisions, campus planners are balancing the health and safety of students, faculty, and staff with other competing factors such as the long-term financial viability and the quality of education of their institutions.

Some operational decisions, such as moving some courses online and reducing capacity in campus housing, may provide a balance between these competing objectives, but they may not be the best practice. According to data collected by The Chronicle of Higher Education [1], around 67\% of the 910 IHEs that were tracked were planning for in-person course instruction as of June $8^{\text {th }}, 2020$. Of the remaining IHEs, $9 \%$ were considering a range of scenarios, $8 \%$ were proposing a hybrid model (a mixture of in-person and online courses), $8 \%$ were planning for online instruction, and $7 \%$ were waiting to decide. As of October $1^{\text {st }}, 2020$, the Chronicle of Higher Education reported that $34 \%$ of nearly 3000 colleges reported being fully online or primarily online, about $27 \%$ were fully in-person or primarily inperson, and another $23 \%$ were operating in a hybrid model.

While weighing decisions on how to deliver courses during the COVID-19 pandemic, student enrollment has weighed heavily on IHE administrators. According to a survey of 192 college and university presidents conducted by the American Council on Education, 78\% strongly agreed that they were concerned about student enrollment due to COVID-19 [2]. In fact, a report from the National Student Clearinghouse Research Center states that undergraduate enrollment in Fall 2020 is $4.0 \%$ below Fall 2019's enrollment and that enrollment of first-time students is down $16.1 \%$ compared to last year [3]. Despite the data, IHEs still have limited knowledge about how to properly plan for campus reopening, including course delivery and campus safety, to maintain enrollment and keep students, faculty, and staff safe. For universities that are using or planning a hybrid mode of course delivery, it is unknown what factors would influence a student to choose in-person or online courses under the current circumstances. These factors may help them better allocate limited academic resources. Furthermore, universities and colleges may want to propose plans that include safety protocols such as social distancing, testing, and masks, but it is unclear whether students will adhere to these protocols or partake in behavior that puts themselves at risk of being infected with and spreading COVID-19.

The objectives of this study were to assess the relative contribution of different attributes related to course modes and safety plans to students' preferences for enrolling or deferring, to analyze stated choices for in-person or online learning during a pandemic, and to estimate students' self-reported and expected compliance behavior related to campus mitigation efforts of COVID-19. We sought to inform decisions around university campuses' modes of course delivery and safety plans during the pandemic and evaluate whether students' enrollment decisions are sensitive to these aspects; to point out what features of students should be considered by IHEs when offering in-person and online courses; and how effective prevention strategies are likely to be given students' compliance behavior. 


\section{Methods}

\subsection{Sample and Study Design}

We conducted an online survey of a sample of 398 college students in the United States from June 25, 2020 to July 22, 2020. Participants were sampled from a medium-sized public university in an urban region in Georgia. Participants received the survey link through an email to their department list-serv. For this survey, we sampled undergraduate students ( $\geq 18$ years old) from an industrial engineering program.

\subsection{Survey Instrument}

The survey asked students about their preferences for enrollment or deferral when presented with combinations of different aspects of campus operations amid the COVID-19 pandemic, and other information like their concern over the pandemic as well as demographic information. The full survey instrument is provided in Appendix C. This survey began with an introduction section which included information that the survey was intended to learn more about the impact of the COVID-19 pandemic on student views related to the reopening of university campuses in Fall 2020. This section also asked about students' levels of concern about COVID-19 as well as questions to gauge their risk attitudes.

The Introduction section was followed by a discrete choice experiment (DCE) designed to elicit student preferences for their university's operations amidst COVID-19 during the Fall 2020 term. DCEs are a subset of conjoint analysis methodologies that are used to elicit stated preferences about decisions that require trade-offs among multiple attributes. They have been commonly used in transportation and marketing research [4], [5], and these methods are also applied to valuation in healthcare [6]. In DCEs, respondents are presented with a series of choice sets, and in each choice set, respondents are asked to choose between 2 or more options (alternatives). Each option is defined by certain characteristics (attributes) which can be assigned different values (levels). The choices made by respondents are then used to infer the importance of each level of the various attributes to those choices.

We identified several important attributes of campus operations by surveying opinion editorials written by university presidents, consultations with undergraduate students, and discussions with members of the COVID-19 campus recovery taskforce at the university where the survey was conducted. After identifying a candidate list of attributes, we chose to vary five attributes in the DCE to limit the cognitive burden on participants [7], and asked about other factors in the remainder of the survey. The final attributes included in our DCE were Mode of Course Delivery, Safety on Campus, Residence Hall Operating Capacity, Tuition Reduction, and Limits on Events and Social Gatherings. Each of these attributes was assigned 4 levels based on the latest recommendations from the Centers for Disease Control and Prevention's interim guidance to institutions of higher education to prepare for COVID-19 [8], as well as discussions with IHE administrators. The attributes and their levels are shown in Table 1. In addition to two unlabeled enrollment options for each choice set, the respondents were also presented with a third option, "Defer enrollment for at least one term". An example question is shown in Figure $\mathrm{A} 1$ in Appendix A.

All discrete choice sets were generated using a fractional factorial design in which all five attributes were considered for each choice set. The design was restricted to eliminate comparisons that included dominated options. In this process, forty-eight choice sets were created, and the design was evaluated based on the D-efficiency statistic to evaluate the balance and orthogonality of the experimental design. 
These choice sets were then evenly and randomly distributed into six blocks, and respondents were randomized to one of the six blocks. The experimental design was created using macros in SAS 9.4 [9] and then the choice tasks were converted to questions in Qualtrics [10] which was used to administer the survey. More details about the design are provided in Appendix B.

After the discrete choice experiment, students were asked about their intended compliance with COVID19 health protocols and their expectations around their classmates' compliance. We also asked a series of other questions related to other preferences around campus operations and perceived risk of COVID19. In addition, the survey included questions about demographic characteristics of the students.

At the time of our survey, students were making their own enrollment and on-campus housing decisions for the Fall 2020 term without full knowledge of the mode of course delivery and details of how housing would operate. These details were announced by their university after the close of this survey.

\subsection{Data Analysis}

The data analysis had three main components. First, we analyzed student responses to estimate expected compliance with health and safety protocols by analyzing student's stated intention to comply with health and safety protocols, as well as students' expectations about whether their classmates would comply or not. Second, we analyzed students' responses for their stated preferences for inperson or online learning. Finally, we inferred the relative importance of the mode of course delivery, safety plans, and tuition reduction on students' choice to enroll or defer through analysis of the responses to the DCE. Additional details are provided below.

\subsubsection{Students' Intentions and Expectations to Comply with Health Protocols}

We analyzed students' responses to questions asking them to state their intended compliance with various health protocols (e.g., mask-wearing, testing, social distancing at gatherings) and their expectations around their classmates' compliance (Section 3 and 4 in Appendix C).

\subsubsection{Students' Choices between In-Person and Online Learning}

We analyzed students' stated choices between learning online compared to learning in-person. First, we analyzed responses to questions that asked students to state whether they preferred an online offering of a course or an in-person offering of a course. The questions specified whether the course was a lecture-based course or a lab-based course (Question 5.5 and 5.6 in Appendix C). We also analyzed responses to questions that asked students whether they preferred to start the semester in-person or to start the semester online given a specific probability that there would be an outbreak on campus which would cause the college to move to entirely online course delivery during the Fall 2020 semester (Question 5.2 and 5.4 in Appendix C). We relied on logistic regressions to explore if there were features that could explain whether a student would prefer the in-person or online option within each scenario and to evaluate the correlations between those features.

All logistic regressions were run in Python using the SciKit-Learn package version 0.23.2 [11] and correlations between features were determined in Python using the Nominal Dython package version 0.6.1. We used dummy coding for all categorical variables in the logistic regression models. Recursive feature elimination was used to determine the most influential features of each logistic regression model. We determined statistical significance from 0 for the coefficients for all logistic regression models using a $p<0.05$ level. 


\subsubsection{Students' Preferences for Enrollment and Deferral using Latent Class Model}

In the DCE block of the survey, we collected student preferences to defer or to enroll in one of two hypothetical alternatives that varied in terms of attributes corresponding to campus operations (i.e., Mode of Course Delivery, Safety on Campus, Residence Hall Operating Capacity, Tuition Reduction, and Limits on Events and Social Gatherings). We analyzed DCE responses to estimate the importance of the different levels of these attributes in terms of their contribution to the utility of alternatives to students, which is represented by the coefficient in a discrete choice model. As a reference, all coefficients of levels of each attribute were assumed to be zeroes for the deferral alternative (giving it zero observed utility). Because the enrollment alternatives were unlabeled, we assumed the coefficients for the levels of each attribute are the same for all enrollment alternatives and estimated them using discrete choice models. We first fitted a model to the entire sample as a single group, and then performed subgroup analyses to examine differences in coefficients across groups described by attitudinal/sociodemographic variables (e.g., current concern level, race) using a conditional logit model. Results are included in Table A1 and Figure A2 - Figure A7 in Appendix A. We determined that there were heterogeneous importance weights and therefore changed to using a latent class model (LCM) to conduct the DCE analysis. The LCM assumes a finite number of classes and allows coefficients of levels of attributes to be the same within each class but different across classes, which therefore helps to identify potential heterogeneous importance placed on the different attributes of the decision to enroll or defer among respondents in a statistical way.

The LCM consists of a class membership model and a class-specific choice model. The class membership model predicts the probability that a given individual belongs to each class, as a function of attributes expected to influence class membership, while the class-specific choice model predicts the probability of choosing each enrollment alternative or the deferral alternative, as a function of the pertinent levels of each attribute and other variables. In our analysis, we first selected all attitudinal/sociodemographic variables which were regarded as being potentially influential in determining respondent's classes and conducted a variable selection process to identify variables that were statistically significant and illustrative of separating classes. We tested several choices for the number of classes in the LCM and iteratively evaluated the resulting models in terms of convergence, fit criteria, and interpretability.

We used dummy coding for categorical variables in all components of the LCM. In the class membership model, ordinal variables were converted into dichotomous variables after investigating whether there were major significant differences between using all levels rather than just two synthesized levels. In the class-specific choice model, three dummy variables were used for each attribute besides Tuition Reduction to represent the non-reference levels so that they can be compared to the reference levels with respect to their relative contributions to the choice outcome. The attribute Tuition Reduction was transformed into Tuition Paid where, for instance, 30\% Tuition Reduction equals $70 \%$ Tuition Paid. The transformed value was then treated as a ratio-scaled variable with just a single coefficient, rather than as an ordinal variable with different coefficients for each level. Thus, the coefficient of this variable can be interpreted as the contribution to the utility of a given alternative that is associated with a one percentage-point increase in tuition paid. The purpose of this transformation was to make it easier to calculate the importance of a given level of an attribute relative to the tuition charge (i.e., in percentage points relative to the regular tuition), and therefore to support comparing the tuition-normalized importance of that level among different classes. We denote this as the "tuition percentage point equivalent" (TPPE). 
Post-estimation analysis included calculating TPPE and class profiles. TPPE was calculated as the ratio of the coefficient of a specific level of an attribute to the coefficient of Tuition Paid in the class-specific choice model. When coefficients of a level in some classes all had the same sign and all of them were statistically significant, we relied on TPPE to identify heterogeneities of importance weights since taking the ratios of coefficients cancelled out scale differences across classes [12]. We investigated each class to determine the average characteristics for members in that class (i.e., class profiles). Expected values of key variables were calculated here, which included several aspects like Stated Concern, Student's SelfReported Intentions to Comply with Health Protocols, Expectations for Classmates' Compliance Behavior, Choice of Course Delivery Mode, Living Situation in Fall 2020, Perceived Risks and Demographic Information (see Appendix C). When we calculated class profiles, we quantified ordinal variables with 5 levels by using integers from 1 to 5 with 1 representing the lowest level and 5 representing the highest level (e.g., 1 = "not at all concerned" and 5 = "extremely concerned" for current concern level, which is Question 1.1 in Appendix C). We quantified binary variables with a 0 or a 1.

All discrete choice analyses were performed in R statistical software version 4.0.2 using the Apollo package version 0.1 .0 [13]. We determined statistical significance from 0 for all coefficients using a $p<$ 0.05 level for the LCM.

\section{Results}

In this section we discuss the results of the survey. We begin by describing the respondents to our survey. Then, we will describe the results from our three sets of analyses. First, we discuss students' stated intentions to comply with health and safety protocols on campus and compare these responses to their expectations for their classmates' compliance with these protocols. Second, we discuss the students' stated preferences for learning in-person or learning online for lab-based and lecture-based classes. We also report our findings about whether students prefer to start the semester in-person or online if there is a chance of outbreaks on campus. Finally, we present the results of the DCE analysis to determine the relative importance of modes of course delivery, safety plans, and tuition to students' decisions to enroll or not.

\subsection{Study Participants}

Among the 1,917 students who were invited to complete the survey, the response rate was $20.8 \% .398$ students participated, and 302 students completed the entire survey. The demographic characteristics of the participating students are shown in Table 2: Characteristics of Student Respondents $(\mathrm{N}=398)$. Comparing the demographic information of the non-respondent group to that of the entire invited cohort, there was slightly less participation among Black / African American students and international students.

\subsection{Students' Intentions and Expectations for Compliance with Health Protocols}

Students reported that they intended to comply with health protocols, as shown in Table 3. However, they were less confident in their classmates' complying with the same protocols. In some cases, students' expectations of their classmates were mostly consistent with their own intentions. $96 \%$ of students stated that they were either extremely likely or somewhat likely to wear a mask in instructional space and around campus if required and $79 \%$ of students believed a majority of their classmates would 
do the same. However, in other cases, students' own intentions were much higher than their stated expectations for their classmates. When masks were recommended but not required, $88 \%$ of students stated that they were extremely likely or somewhat likely to wear a mask, but only $37 \%$ of students believed that almost all or a majority of their classmates would wear them.

There were also large discrepancies in students' own intentions and their expectations for their classmates regarding social gatherings. $74 \%$ of students stated they would comply with a policy to limit social gatherings to 20 people. By contrast, $49 \%$ of students believed that most of their classmates would not follow this rule. When asked about practicing social distancing at social gatherings, $54 \%$ of students stated they were extremely likely or somewhat likely to maintain 6 feet of distance. However, $68 \%$ of students believe that most of their classmates would not follow this protocol.

\subsection{Students' Choices between In-Person and Online Courses}

Table 4 presents students' stated choices between in-person and online learning. When there is no assumption regarding future outbreaks on campus during Fall $2020,47 \%$ of respondents stated that they would choose an in-person mode of instruction for lecture-based courses, and $60 \%$ of respondents chose the in-person instruction for lab-based courses.

We estimated logistic regression models of the choice between in-person and online course delivery separately for lecture-based and lab-based courses, and we reported our results in the first two columns of Table 5. We present the statistically significant odds ratios, i.e., the factors by which the odds of choosing in-person over online course delivery changed with respect to the presence of (or one-unit increase in) the associated feature. For lecture-based courses, the logistic regression model shows statistical significance of the association between current concern level, perceived risk of infection, current living suitability for online courses, risk-seeking and birthyear (Questions 1.1, 7.3, 6.6, 1.3, 8.2 in Appendix C, respectively) and choosing an online lecture-based course. Specifically, the results of the model indicate that:

i. students with greater current concern level are more likely to choose online courses than students with lower concern levels,

ii. students with higher perceived risk of infection are more likely to choose online courses than those with lower perceived risk of infection,

iii. students with better current living suitability for online courses are more likely to choose online courses than students with worse current living suitability for online courses,

iv. students who are more risk-seeking are more likely to choose in-person courses than students who are less risk-seeking, and

v. younger students are more likely to choose in-person courses than older students.

For lab course preferences, we see the current concern level and current living suitability for online courses variables both are not found to be significant but the other three indicators are still significant in the same direction as for lecture-based courses.

Table 4 also presents students' choices between starting the semester in-person or starting the semester online when there is a given probability of a future outbreak that would cause the campus to move entirely online during Fall 2020. When there was a 50\% chance of a future outbreak that would cause campus to shut down and move to entirely online instruction (Question 5.2 in Appendix C), we found that $56 \%$ of students preferred starting the semester in-person. However, in the scenario where 
there was a $100 \%$ chance of this severe outbreak (Question 5.4 in Appendix C), only 36\% of respondents preferred starting the semester with in-person courses.

Student responses to current concern level, perceived risk of infection, perceived risk of adverse effects if infected and current living suitability for online courses were summarized in Table 4 . These factors together with risk-seeking, and birthyear appeared as influential to students' choice between in-person vs. online mode, as summarized in Table 5. The last two columns of Table 5 show the factors that are influential to students' choices between the in-person mode and the online mode of lecture-based courses when a probability of future severe outbreaks is given. When presented with a $50 \%$ chance of a severe outbreak, students with high perceived risk of infection and high perceived risk of adverse effects if infected (Question 7.4 in Appendix C) were more likely than others to choose the online mode of instruction. Younger and more risk-seeking students were more likely than others to choose to start the semester in-person. When presented with a $100 \%$ chance of a severe outbreak, students with a higher perceived risk of infection and perceived risk of adverse effects if infected as well as students whose current living suitability was conducive to online learning were more likely than others to prefer online courses. Younger students were more likely than others to prefer in-person courses in this scenario.

\subsection{Students' Preferences for Enrollment and Deferral Influenced by Course Modes and Safety Protocols}

From our analysis of DCE, we determined the best LCM was a three-class model with variables current concern level and choice of lecture-based courses (Questions 1.1 and 5.5 in Appendix C) included in the class membership model. We accepted this model due to its fit criteria (i.e., AIC, BIC and adjusted $\rho^{2}$ ) and because the model offered the best interpretability and convergence among all candidate models with which we experimented. Table 6 and Figure 1 present the final estimated model with AIC of 4616.7, BIC of 4906.4 , and adjusted $\rho^{2}$ of 0.32 (equally-likely base). The fit results of LCMs with 2, 3, or 4 classes are shown in Table A2 in the Appendix A. We did not investigate LCMs with 5 or more classes because the estimations of LCMs with 4 classes was poor.

\subsubsection{Class Membership Model}

The class membership model differentiated three potential classes, with both current concern level and choice of lecture-based courses being significant. Among all students, those who had the most concern and the strongest preference for online lecture-based courses had highest probabilities of belonging to Class 3. Those who were moderately concerned and preferred online lecture-based courses were more likely to belong to Class 2, while those who were not-so-concerned and did not prefer online lecturebased courses were more likely to belong to Class 1 . To distinguish these classes, we refer to Class 1 as "low-concern" students, Class 2 as "moderate-concern" students, and Class 3 as "high-concern" students.

The class probabilities gave insights into the distribution of students' attitudes towards the COVID-19 pandemic. According to Table 6, 29\% of students fell into Class 1 being not-so-concerned while 17\% fell into Class 3 being highly concerned. Most of the students were in the "moderate-concern" class (i.e., there was an average $54 \%$ probability of belonging to Class 2 ). The class membership probabilities are consistent with the reported current concern level of students in Table 4 which found $14 \%$ of students were "not at all concerned" or "slightly concerned", 22\% were "extremely concerned" and 64\% were "moderately concerned" or "very concerned". 
We suspected that students planning to live on campus in Fall 2020 had different preferences for Residence Hall Operating Capacity than students living off campus, so we also tried to consider living location in Fall 2020 (Question 6.7 in Appendix C) in the class membership model. The results showed that living location in Fall 2020 was not significant in the class membership model for all three classes, as was also the case for 2-class and 4-class models.

\subsubsection{Choice Models}

We observed that the choice models differed substantially among the 3 latent classes, suggesting that an LCM was a reasonable choice. In particular, the fact that in several instances the same feature had a significantly positive coefficient for at least one class and a significantly negative coefficient for at least one class highlights the extent of the heterogeneity in our sample. If we only used a single-class model, such features may not have even appeared to be significant, given the estimation of a sample-wide "average" coefficient that may have been close to 0 (e.g., comparing the coefficient for the level "20 people" of Limits on Event and Social Gatherings in Table 6 and Table A1).

For the LCM, we present the class membership model and class-specific choice model in Table 6. TPPE was calculated for all non-reference levels of attributes, as a supplement to better distinguish the heterogeneous importance weights of levels in different classes (see Table 7). TPPE can be interpreted as the contribution of a given level of an attribute (relative to its reference level) to the utility of an alternative, in terms of a percentage-point change in Tuition Paid. For instance, for Class 1, we observe that the Tuition Paid coefficient is -0.013 . That is, a percentage point increase in tuition reduces utility by 0.013 utiles. The "Masks recommended \& some COVID-19 testing" level of the Safety on Campus attribute has a coefficient of 0.654 , meaning that if campus safety requirements change from the reference level of "No masks and no testing" to "Masks recommended \& some COVID-19 testing", the utility increases by 0.654 utiles for Class 1 students. The ratio of the latter to the former, which is the TPPE for level "Masks recommended \& some COVID-19 testing", is around 50 as shown in the corresponding entry of Table 7. TPPE gives the trade-off between a specific level and Tuition Paid and represents the (maximum) additional percentage points of tuition that a student is willing to pay for obtaining an improvement in that attribute level and not be worse off than before (or, alternatively, the minimum percentage-point reduction in tuition that a student would require if an attribute dropped to a less desirable level). Expressed in the most natural way, this result indicates that a Class 1 (low concern) student would require a 50 percentage-point reduction in tuition to be equally well off if the Safety on Campus attribute worsened from "Masks recommended \& some COVID-19 testing" to "No masks and no testing". As previously stated in Section 2.3.3 since calculating TPPEs cancels out scale differences we can use TPPEs to compare the importance of the various levels of attributes to students' preferences for enrollment and deferral across classes. For example, we note that Class 3 (high concern) students would require a larger ( 83 percentage-point) reduction in tuition than Class 1 to be equally well off under the same change to Safety on Campus.

The alternative specific constants (ASCs) indicate if students preferred the base alternative formed by all reference levels of all attributes to the "Defer enrollment for at least one term" alternative, whose utility to students was assumed to be 0 in this study. As indicated by Table 6 , when all attributes are at their reference levels, students in Class 1 or Class 2 had stronger preferences for enrolling in the Fall 2020 semester than students in Class 3 since those two classes had positive and significant ASCs. TPPE results further illustrated that the "low-concern" students of Class 1 had the strongest preference for 
taking courses in the Fall 2020 semester with the base alternative since its TPPE for ASC is the largest among all three classes.

The coefficients for levels of the attribute Mode of Course Delivery represent the importance of course delivery modes that incorporate online portions of classes compared to the reference mode "All classes delivered entirely in-person" on the decision to enroll or not. Based on results shown in Table 6 and Table 7, students in more concerned classes (i.e., Class 2 and Class 3) placed more importance on modes incorporating at least some online courses. However, students in Class 1 did not like having online courses compared to completely in-person courses. Students in the "high-concern" class (Class 3) put much more importance on entirely online courses when deciding to enroll compared to students in the "moderate-concern" class (Class 2), indicated by the result that the TPPE of "All courses delivered entirely online" in Class 3 was more than twice as large as the corresponding TPPE in Class 2.

For each class of students, placing some safety measures like requirements for masks and testing on campus was better than "No masks \& no testing". The importance weight of Safety on Campus increased from level "Mask required \& extensive COVID-19 testing" to level "Masks recommended \& some COVID-19 testing" for Class 1 students. On the contrary, the more concerned students in Class 2 or Class 3 placed more importance on stricter safety measures. "High-concern" students of Class 3 placed the most importance on any level of Safety on Campus, as indicated in Table 7.

For attributes Residence Hall Operating Capacity and Limits on Events and Social Gatherings, having more coefficients be statistically insignificant in Table 6 suggested they were not as important as Mode of Course Delivery and Safety on Campus to influencing students' choices between enrolling or deferring. Those who were low-concern (Class 1) exhibited a substantial disutility when limiting the operating capacity of residence hall or the number of people for events and social gatherings, especially for the strictest limits (i.e., closing residence hall and no more than 20 people for events and social gatherings). "Moderate-concern" students (Class 2) placed the most importance on having $25 \%$ capacity of residence hall with no roommates and no shared bathrooms and placed the most importance on all limits on events and social gatherings. Class 2 students did not show a significant importance weight for having $50 \%$ capacity of residence hall with no roommates but shared bathrooms and for closing the residence hall. All coefficients of levels of Residence Hall Operating Capacity and Limits on Events and Social Gatherings were insignificant for Class 3 students.

The choice model for each class can be used to estimate the probability that students in each class would choose to enroll rather than defer under specified levels of attributes. We tested several hypothetical scenarios to check enrollment probabilities of students in our DCE. The first is a "businessas-usual" scenario in which courses are delivered entirely in-person, no requirement on mask-wearing and no testing, $100 \%$ operating capacity for residence halls, full tuition, and no limit on the size of social gatherings. Under a "business-as-usual" scenario, the low-concern class (Class 1 ) was predicted to enroll with $98.9 \%$ probability and the "moderate-concern" class (Class 2 ) was predicted to enroll with $94.8 \%$ probability. However, the "high-concern" class (Class 3 ) was predicted to enroll with only $17.0 \%$ probability. Weighting by the class membership probabilities, the weighted average enrollment probability is $82.8 \%$ for this "business-as-usual" scenario. In contrast, another tested scenario is a "completely online" scenario in which a $5 \%$ tuition reduction is given and has weighted average enrollment probability of $94.6 \%$ with the low-concern class enrolling with $85.4 \%$ probability, the moderate-concern class enrolling with $99.7 \%$ probability, and the high-concern class enrolling with 
93.9\% probability. A scenario with a higher enrollment probability is a "strict on-campus hybrid" scenario in which large courses are delivered online with small courses delivered in-person, required mask-wearing and extensive testing, residence halls are at $25 \%$ capacity (in which there are no roommates and no shared bathrooms), no tuition reduction, and a limit of 20 people at social gatherings. This "strict on-campus hybrid" scenario has a weighted average enrollment probability of $97.6 \%$ because it broadly appeals to students from the different classes: low-concern class are expected to enroll with $94.7 \%$ probability, the moderate-concern class has a near $100 \%$ probability of enrolling, and the high-concern class has a $95.1 \%$ probability of enrolling.

\subsubsection{Class Profiles}

Table 8 presents class profiles for all three latent classes. All variables associated with Stated Concern and Perceived Risks showed the differences expected across classes. From Class 1 to Class 3, the expected values of current concern level, current concern versus concern in mid-March (Question 1.2 in Appendix C), perceived risk of infection (Question 7.3 in Appendix $\mathrm{C}$ ) and perceived risk of adverse effects if infected (Question 7.4 in Appendix C) increased while expected values of risk seeking (Question 1.3 in Appendix C) decreased. This phenomenon indicated that students in the two more concerned classes had more current concern, more concern compared to mid-March, and more perceived risks than students in Class 1. At the same time, "high-concern" and "moderate-concern" students were more likely to exhibit risk aversion while "low-concern" students were more likely to be risk-seeking.

The responses for Student's Self-Reported Intentions to Comply with Health Protocols (Section 3 in Appendix C) and Expectations for Classmates' Compliance Behavior (Section 4 in Appendix C) followed the same trend within each class, but the magnitude of average responses varied across classes slightly. Students in Class 2 or Class 3 were more likely to intend to comply with safety practices of requirements for masks, testing, safety protocol, and staying home if having a fever or other concerning symptoms than "low-concern" Class 1 students, even though all of them stated they were at least somewhat likely to engage in those practices. Students' stated engagements in limits on social gatherings and practicing social distancing at social gatherings were not as high as that of other practices, especially for their selfreported intentions to practice social distancing at social gatherings (social distancing (self), Question 3.7 in Appendix C). For all 3 classes, the expected values of social distancing (self) were around 3, the value representing the middle level of each 5-level ordinal variable (see Section 3.2). "Low-concern" students were a little bit more positive about their Expectations for Classmates' Compliance Behavior towards those same practices used to gauge their self-reported intentions than "moderate-concern" and "high-concern" students. Similar to self-reported intentions for limits on social gatherings and for practicing social distancing at social gatherings, on average students did not think their classmates were likely to engage in these two practices.

Class 2 and Class 3 students were much more inclined to choose online courses, either for lecture-based courses or for lab-based courses (Question 5.6 in Appendix C), than the "low-concern" Class 1 students. The result that the expected values of both choices are the highest for "high-concern" students was consistent with the results we generated from class-specific choice models. Since more concerned students might think delivering lab-based courses online could not guarantee acceptable quality, the expected values of this choice were less than that of the choice of lecture-based courses in Class 2 and Class 3. 
Results also suggested that students in the "moderate-concern" class or "high-concern" class had slightly better current living suitability for online courses (Question 6.6 in Appendix C) and were more likely to live off campus in Fall 2020 (Question 6.7 in Appendix C).

We also compared the expected values of sociodemographic variables for members of each class and found differences among the classes in terms of race, political leaning and world view. Asian / Pacific Islander students were more likely to be in Class 2 or Class 3, while White / Caucasian students were more likely to be in Class 1 . Our initial experiments with subgroup analysis also revealed that Asian / Pacific Islander students were generally more concerned about the COVID-19 pandemic than White / Caucasian students (see Figure A7 in Appendix A). The differences among classes of shares of Black / African American and Hispanic / Latino students were not significant, which could be attributed to the overall low shares of respondents belonging to these two races. Students in the "moderate-concern" class or "high-concern" class tended to politically lean Democrat and had a more liberal world view. "Low-concern" students were inclined to lean Republican and had a more conservative world view.

\section{Discussion}

The purpose of this study was to examine students' willingness to comply with health protocols on campus, choices between in-person and online learning, and the importance of course modes and safety plans to them when deciding to enroll in institutions of higher education amid the COVID-19 pandemic. In contrast to many surveys of students done at the beginning of the summer of 2020 when cases in Georgia remained under 1,000 new cases per day, students completed this survey when cases started to dramatically rise. On the first day that the survey was open, the state of Georgia saw 1,900 new cases of COVID-19 and the number of new cases grew throughout the survey period with 3,875 new cases on the final day of the survey. On June 29, the governor of Georgia signed two executive orders that extended the state's Public Health State of Emergency and its existing COVID-19 safety measures.

During the time of our survey, students were having to make their own enrollment and on-campus housing decisions without full knowledge of the mode of course delivery. Students completed the survey by July $15^{\text {th }}, 2020$ and the deadline to defer enrollment at this institution was July $24^{\text {th }}, 2020$ at the beginning of the survey. While the survey was in progress, the deadline to defer was delayed until August $3^{\text {rd }}, 2020$. Students were also weighing their housing options, because students who had previously opted for on-campus housing had until June $30^{\text {th }}$ to cancel their housing contract. Students were given the option to defer their housing deposit to a later semester if their deferral request was received by August $3^{\text {rd }}$. On July $20^{\text {th }}$ (after the survey had ended), the institution revealed the course modality to students; modes were in-person, online, or hybrid and varied by class section.

Although there have been several surveys of university students during the COVID-19 pandemic, many have focused on the influence of the transition to online learning. They have investigated students' satisfaction with the online mode, compared students' concern over financial stability, academic demands, health and well-being before and after the transition, and explored students' plans to enroll in the Fall 2020 semester [14]-[20]. These surveys found that students were more satisfied with the inperson mode before the transition. Even though students were more concerned about almost all aspects related to their academic lives, most of them still planned to enroll in Fall 2020. Perhaps the most closely related work to ours is a survey conducted among 46 Columbia University public health students [21] which used a "standard gamble" [22] exercise to measure students' risk tolerance for inperson mode and for social gatherings, and estimated the proportion of tuition they were likely to pay 
for entirely online courses. That study found that students would accept a $23 \%$ risk of infection to get the in-person mode, pay $48 \%$ of typical tuition for online courses, on average; and $41 \%$ of them would attend social gatherings even with some risks of infection. In contrast to existing surveys, our research is the only one, to our knowledge, to elicit students' importance weights for different aspects of campus recovery operations with a discrete choice experiment, to evaluate features influencing students' choices between in-person and online learning, and to examine students' intentions and expectations around compliance with health protocols.

The results of our DCE indicated that there were heterogenous importance weights for different features of campus safety measures and educational plans when deciding whether to enroll. Most students fell into a moderately concerned group. These students were strongly likely to enroll so long as courses were not delivered entirely in-person and there were sufficient safety measures in place around mask-wearing and testing and limits for social gatherings.

We found there was a latent class of students who were highly concerned, comprising about $17 \%$ of students. Students in this highly concerned class placed the most importance on an entirely online mode of course delivery and for the strictest requirements for masks and COVID-19 testing. Stricter safety measures and more online courses would increase the probability that "high-concern" students enrolled in Fall 2020. Surprisingly, the importance of limits on social gatherings was not significant for this class of student, which could be attributed to the dominant importance of the entirely online mode and the design of DCE (see details in Appendix B).

In contrast, there was another latent class of students who were not very concerned and comprised about $30 \%$ of the students. Students in this class placed importance on entirely in-person classes to online classes and on only modest levels of safety measures around mask-wearing and testing, limits for residence hall and social gatherings. Stricter limits would decrease their utility of enrolling.

Our DCE analysis offered some insights for administrators when creating plans for campus operations during a pandemic. We found that scenarios that offered an on-campus experience with large classes delivered online and small classes delivered in-person, strict safety protocols in terms of mask-wearing, testing, and residence halls, and any limits on the size of social gatherings were broadly the scenarios with the highest expected enrollment. These scenarios tended to appease all "low-concern", "moderateconcern", and "high-concern" students. Based on our DCE results, recommending or requiring maskwearing and conducting some COVID-19 testing on campus increases the probabilities of enrolling for all students. Since policies around testing and mask-wearing apply to all students, campus administrators may wish to meet preferences of as many students as possible and most students were quite sensitive to whether safety measures are put in place. Restricting the number of students in campus housing without closing residence halls was preferred by most students. In addition, modest limits on the sizes of social gatherings were also largely preferred. When deciding whether to offer courses in an entirely online format, in-person format, or a hybrid format, campus administrators should be aware of the heterogeneous preferences among students and that a one-size-fits-all approach may not satisfy large portions of the student population. Most students stated they were less likely to enroll when all classes were delivered entirely in-person while any degree of online classes was not preferred by the not very concerned students. Therefore, to properly take care of the needs of all students, administrators may better consider providing choices of different course modes to students if there are resources available to do so.

According to our analysis of students' choices between in-person and online learning, students' perceived risks of infection of COVID-19, risk seeking level, birthyear, and current living suitability with respect to online learning are the most influential factors for their decisions under most conditions with 
or without assumed probabilities of future outbreaks. Students who perceived themselves at higher risk of infection, were risk-seeking, were older, and had better living suitability were more likely to choose online learning than others. We found that students tended to prefer in-person instruction for lab-based courses but online instruction for lecture-based courses, which suggested IHE administrators might still need to offer lab-based courses in-person but with sufficient safety measures implemented at the same time. Younger students, especially incoming freshmen, were less worried about the COVID-19 pandemic and thus consistently preferred in-person learning. This phenomenon highlighted the needs of universities to conduct awareness campaigns for information and impacts of COVID-19 among new students and at least encourage them to take some precautions when taking courses in-person.

In terms of self-reported compliance and expectations for compliance of classmates for various health protocols, students tended to report higher likelihood of complying for themselves than expectations for their classmates. There are a few possible explanations for these discrepancies. There may be social desirability bias (respondents overreport their own compliance, but more accurately capture it in their reported perceptions of others' compliance) [23], relative superiority bias (respondents assume others are less "virtuous" than they themselves are in this respect) [24], and/or non-response bias (those who think the whole "pandemic thing" is overblown may be less likely to respond to the survey altogether). Presumably some sort of bias is indicated, or the aggregate statistics for the respondents themselves would roughly match the aggregate statistics they report to be the case for the population (of fellow students) at large.

Despite these discrepancies, there are still some valuable insights that can be gained from these responses. In election forecasting, there is evidence that expectations tend to yield better estimates than intentions [25]. If the same holds, students' expectations of their classmates may be a better predictor of expected compliance to those health protocols than students self-reported compliance behavior. While students expected their classmates and themselves to be willing to comply with some safety measures (e.g., wearing masks if required and staying home from class if concerning symptoms arise), students were not optimistic about their classmates' compliance with other protocols regarding social gatherings that are suggested by the Centers for Disease Prevention and Control (i.e., limiting social gathering size to under 20 people, maintaining 6 feet of distance at gatherings [26]), which should elicit the awareness of universities. Since it was reported that students' gatherings can be blamed for campus COVID-19 outbreaks in Fall 2020 [27], universities might need to figure out an effective way to urge students to be more active in limiting the size of social gatherings and practicing social distancing.

Several limitations of our study should be kept in mind. First, our response rate was about $21 \%$, and there was likely to be a non-response bias. We suspect that students who thought the COVID-19 pandemic was not a pressing issue would be less likely to respond than students who thought the pandemic was an important issue. Therefore, we may be seeing more representation of the "moderateconcern" and "high-concern" students than "low-concern" students.

Second, students surveyed in the study were all industrial engineering students at a medium-size university in an urban area. Students from other majors and other types of universities may not share the same preferences and opinions, especially since industrial engineering majors do not have as heavy a requirement for lab classes that are pedagogically better delivered in person.

Third, the university from which students were surveyed had announced its campus reopening plans on June 17, 2020. The plan did not require face coverings in instructional areas, motivating a petition among the students and faculty at this university to require face coverings in instructional spaces on campus. The decision to mandate masks was announced on July 6,2020 , which overlaps with the period 
during which the survey was open. Therefore, the responses to this survey may be a reaction to this proposed plan and the forced changes.

Fourth, during the survey, the United States Immigration and Customs Enforcement (ICE) agency announced on June $6^{\text {th }}, 2020$ that the Student and Exchange Visitor Program would modify the temporary exemption that allowed nonimmigrant students to take online classes due to the pandemic, meaning that students whose universities went completely online could have faced deportation. This may have influenced the responses around preferences for online vs. in-person classes. On June $14^{\text {th }}$, 2020, ICE announced that it was reversing that decision.

Finally, these stated preferences were gathered before the Fall 2020 semester. Preferences of students for Spring 2021 may have changed after their experience during Fall 2020 in which they now have a better sense of what the course delivery modes mean in practice and what the safety measures on campus look like in their daily life.

In summary, the existence of heterogeneous preferences makes designing a one-size-fits-all plan for reopening campuses difficult. Campus administrators should try to offer flexibility in their campus recovery policies, especially for those aspects largely influencing students' decisions, like the mode of delivery for courses. For instance, providing a choice between an online offering and an in-person offering of courses or having an instructor record live lectures for remote viewing could be good ways to appeal to more students but these approaches would undoubtedly require instructors or resources for delivering both formats. There are other options that also satisfy heterogenous preferences of many students, such as offering large courses online and optional small group activities in-person in which masks and COVID-19 surveillance tests are required. At the same time, universities should be aware that students may not comply with health protocols around social gatherings and therefore better prepare some safety precautions for emergencies in advance.

All these findings suggest that surveying students about their perceived importance of course delivery and comfort with the proposed safety plans may help inform whether students plan to enroll at their institutions of higher education during a pandemic. Further, surveying students about the trade-offs between different educational and safety aspects of reopening campuses can help campus administrators design plans that balance the needs of their students and at the same time improve the overall likelihood of student enrollment. 


\section{Acknowledgements}

Funding/Support: This work was supported by a grant from the Thos and Clair Muller Research Endowment Fund of the Georgia Institute of Technology's H. Milton Stewart School of Industrial and Systems Engineering, and a grant from the Georgia Institute of Technology Executive Vice President for Research COVID-19 Rapid Response Seed Grant Program.

Role of the Funder/Sponsor: The sponsor did not influence the design or analysis of this study.

Additional Contributions: We thank Laurie Garrow, PhD, Co-Director of the Center for Urban and Regional Air Mobility (CURAM) at Georgia Tech for her assistance with the initial design of the survey and discrete choice experiment. 


\section{References}

[1] Chronicle Staff, "Here's a List of Colleges' Plans for Reopening in the Fall," The Chronicle of Higher Education, 2020. .

[2] M. Taylor, J. Turk, and C. Sanchez, "College and Unviersity Presidents Respond to COVID-19: May 2020 Survey," 2020.

[3] "COVID-19: Stay Informed - National Student Clearinghouse Research Center." [Online]. Available: https://nscresearchcenter.org/stay-informed/. [Accessed: 08-Dec-2020].

[4] L. A. Garrow, Discrete choice modelling and air travel demand: theory and applications. Routledge, 2016.

[5] P. L. Mokhtarian and X. Cao, "Examining the impacts of residential self-selection on travel behavior: A focus on methodologies," Transp. Res. Part B Methodol., vol. 42, no. 3, pp. 204-228, 2008.

[6] M. Ryan, K. Gerard, and M. Amaya-Amaya, Using discrete choice experiments to value health and health care, vol. 11. Springer Science \& Business Media, 2007.

[7] G. A. Miller, "The magical number seven, plus or minus two: Some limits on our capacity for processing information.," Psychol. Rev., vol. 63, no. 2, p. 81, 1956.

[8] Centers for Disease Control and Prevention, "Interim Guidance for Administrators of US Institutions of Higher Education." .

[9] W. F. Kuhfeld, "Experimental design, efficiency, coding, and choice designs."

[10] S. Weber, "A Step-by-Step Procedure to Implement Discrete Choice Experiments in Qualtrics," Soc. Sci. Comput. Rev., p. 0894439319885317, 2019.

[11] F. Pedregosa et al., "Scikit-learn: Machine Learning in Python," J. Mach. Learn. Res., vol. 12, no. 85, pp. 2825-2830, 2011.

[12] G. Hoetker, "The use of logit and probit models in strategic management research: Critical issues," Strategic Management Journal, vol. 28, no. 4. John Wiley \& Sons, Ltd, pp. 331-343, 01Apr-2007.

[13] S. Hess and D. Palma, "Apollo: A flexible, powerful and customisable freeware package for choice model estimation and application," J. Choice Model., vol. 32, Sep. 2019.

[14] "University of Maryland, Baltimore Survey of Student COVID-19 Experience - June 2020." [Online]. Available:

https://webcache.googleusercontent.com/search?q=cache:K8GWOUZQYncl:https://www.umary land.edu/media/umb/af/ira/surveys/2020-Student-COVID-19-Survey-Briefing-FINAL072420. pdf+\&cd=1\&hl=en\&ct=clnk\&gl=us. [Accessed: 08-Dec-2020].

[15] "COVID-19 Current College Students Survey Full Report | Carnegie Dartlet." [Online]. Available: https://www.carnegiedartlet.com/resources/covidreport-currentstudents/. [Accessed: 08-Dec2020].

[16] "Suddenly Online: A National Survey of Undergraduates During the COVID-19 Pandemic." 
[Online]. Available: https://digitalpromise.dspacedirect.org/handle/20.500.12265/98. [Accessed: 08-Dec-2020].

[17] California Student Aid Commission \& University of California and California Education Lab, "COVID-19 student survey, California Student Aid Commission," California Student Aid Commission, 2020.

[18] K. M. Soria, “Graduate and Professional Students' Fall 2020 Re-Enrollment Plans,” 2020.

[19] K. M. Soria, “Graduate and Professional Students' Financial Hardships During the COVID-19 Pandemic: Evidence from the gradSERU COVID-19 Survey." 2020.

[20] Student Experience in the Research University (SERU) Consortium, "Will Students Come Back? Undergraduate Students' Plans to Re-Enroll in Fall 2020," 2020.

[21] Z. Zafari, L. Goldman, K. Kovrizhkin, and P. Muennig, "Willingness to Pay Tuition and Risk-Taking Proclivities Among Students During the Covid-19 Pandemic: A Fundamental Conundrum for Universities," Nov. 2020.

[22] P. H. Farquhar, "Utility Assessment Methods.," Manage. Sci., vol. 30, no. 11, pp. 1283-1300, Nov. 1984.

[23] R. J. Fisher, "Social Desirability Bias and the Validity of Indirect Questioning," 1993.

[24] B. Headey and A. Wearing, "The sense of relative superiority - Central to well-being," Soc. Indic. Res., vol. 20, no. 5, pp. 497-516, Oct. 1988.

[25] D. Rothschild and J. Wolfers, "Forecasting elections: Voter intentions versus expectations," Available SSRN 1884644, 2011.

[26] "Social Distancing." [Online]. Available: https://www.cdc.gov/coronavirus/2019-ncov/preventgetting-sick/social-distancing.html. [Accessed: 21-Dec-2020].

[27] "Coronavirus: Fraternities blamed for campus outbreaks as universities struggle to keep students in class." [Online]. Available: https://www.cnbc.com/2020/09/09/coronavirus-universitiesblame-greek-life-for-campus-reopening-headaches-were-having-a-significant-issue.html. [Accessed: 08-Dec-2020]. 
Tables and Figures

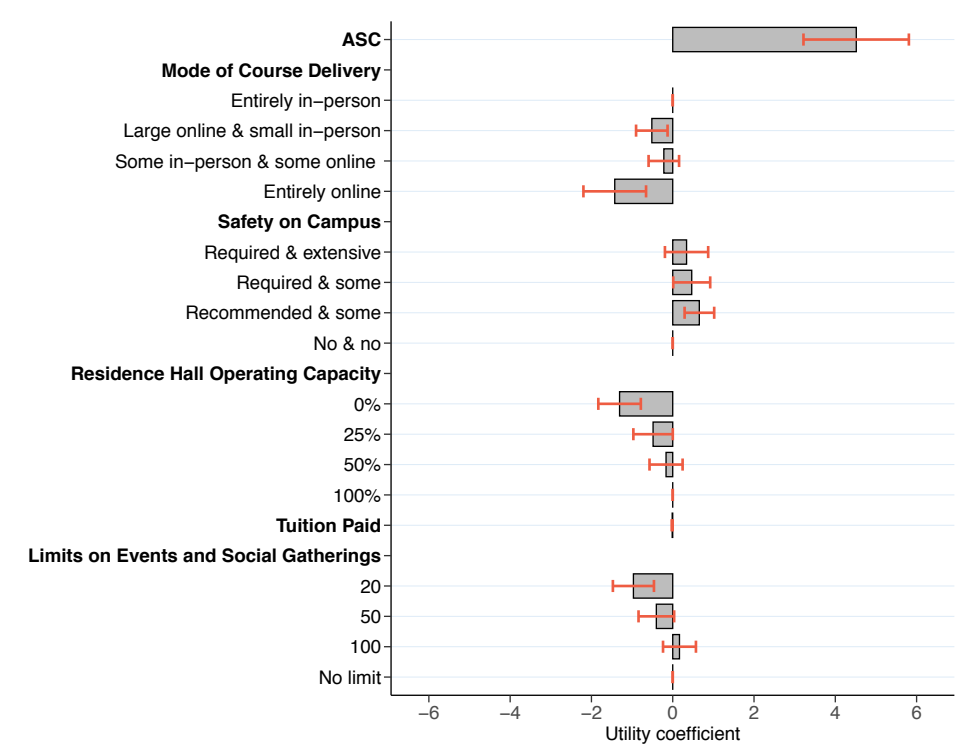

(a) Class 1: "Low-concern" student

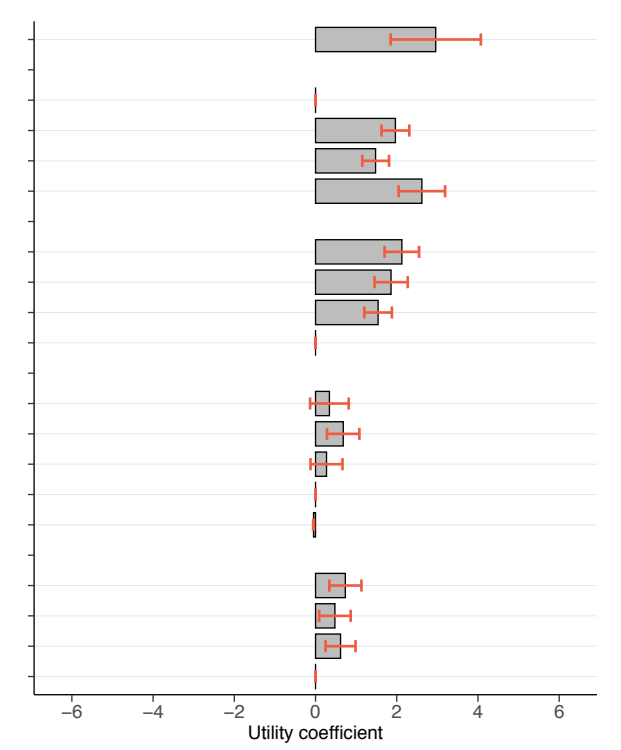

(b) Class 2: "Moderate-concern" student

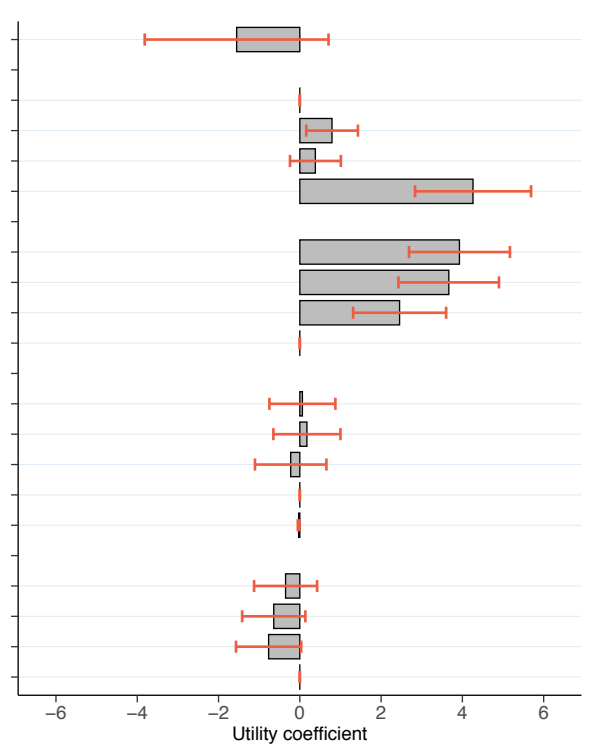

(c) Class 3: "High-concern" student

Figure 1: Estimated results of final latent class model ( $N=386)$; (a) Class 1: "Low-concern" student (b) Class 2: "Moderate-concern" student (c) Class 3: "High-concern" student 


\begin{tabular}{ll}
\hline Attributes & Levels \\
\hline Mode of Course Delivery: & All classes delivered entirely in-person* \\
& All classes deliver large lectures online \& small group activities in- \\
& person \\
& Some classes delivered entirely in-person \& some classes \\
& delivered entirely online \\
& All classes delivered entirely online \\
& Masks required \& extensive COVID-19 testing \\
& Masks required \& some COVID-19 testing \\
Safety on Campus: & Masks recommended \& some COVID-19 testing \\
& No masks \& no testing* \\
& Closed, 0\% \\
& Open, 25\% capacity (no roommates \& no shared bathrooms) \\
Residence Hall Operating & Open, 50\% capacity (no roommates, but shared bathrooms) \\
Capacity: & Open, 100\% capacity* \\
& None* \\
Tuition Reduction: & $10 \%$ \\
& $20 \%$ \\
& $30 \%$ \\
& 20 people \\
Limits on Events and Social & 50 people \\
Gatherings: & 100 people \\
& No limit* \\
& \\
& \\
& \\
& \\
& \\
&
\end{tabular}

* Reference level 


\begin{tabular}{ll}
\hline Characteristic & No (\%) \\
\hline Female & $201(50.50 \%)$ \\
Age (year) & \\
$18-19$ & $112(28.14 \%)$ \\
$20-21$ & $153(38.44 \%)$ \\
$22-23$ & $108(27.14 \%)$ \\
$24-30$ & $18(4.52 \%)$ \\
$>30$ & $0(0.00 \%)$ \\
Did not answer & $7(1.76 \%)$ \\
Race/ethnicity & \\
Asian / Pacific Islander & \\
Black / African American & $144(36.18 \%)$ \\
Hispanic / Latino & $11(2.76 \%)$ \\
Native American & $47(11.81 \%)$ \\
White / Caucasian & $1(0.25 \%)$ \\
Other & $220(55.28 \%)$ \\
Academic standing as of Fall 2020 & $5(1.26 \%)$ \\
Freshman & \\
Sophomore & \\
Junior & $61(15.33 \%)$ \\
Senior & $54(13.57 \%)$ \\
Did not answer & $92(23.12 \%)$ \\
International student & $189(47.49 \%)$ \\
Receiving financial aid & $2(0.50 \%)$ \\
Highest level of parents' education & $59(14.82 \%)$ \\
Some grade/high school & $213(53.52 \%)$ \\
Completed high school or GED & \\
Some college/technical school & $8(2.01 \%)$ \\
Bachelor's degree & $17(4.27 \%)$ \\
Some graduate school & $20(5.03 \%)$ \\
Completed graduate degree(s) & $133(33.42 \%)$ \\
\hline & $22(5.53 \%)$ \\
& $194(48.74 \%)$ \\
\hline$d$ not answer & $4(1.01 \%)$ \\
\hline
\end{tabular}


Table 3: Students' stated likelihood of complying with health protocols compared to the expected compliance of their classmates ( $N=396)$.

\begin{tabular}{|c|c|c|c|c|}
\hline Safety Protocol & How likely are you? & $\%$ & $\begin{array}{l}\text { Percentage of } \\
\text { classmates? }\end{array}$ & $\%$ \\
\hline \multirow{5}{*}{$\begin{array}{l}\text { Wear a cloth face covering while moving } \\
\text { around campus and in instructional } \\
\text { space if required }\end{array}$} & Extremely likely & 88.7 & Almost all & 31.7 \\
\hline & Somewhat likely & 7.8 & A majority & 47.0 \\
\hline & $\begin{array}{l}\text { Neither likely nor } \\
\text { unlikely }\end{array}$ & 1.2 & About half & 15.0 \\
\hline & Somewhat unlikely & 0.5 & A minority & 4.3 \\
\hline & Extremely likely & 1.8 & Almost none & 2.0 \\
\hline \multirow{5}{*}{$\begin{array}{l}\text { Wear a cloth face covering while } \\
\text { moving around campus and in } \\
\text { instructional space if recommended, but } \\
\text { not required. }\end{array}$} & Extremely likely & 67.6 & Almost all & 8.3 \\
\hline & Somewhat likely & 20.1 & A majority & 29.2 \\
\hline & $\begin{array}{l}\text { Neither likely nor } \\
\text { unlikely }\end{array}$ & 5.3 & About half & 38.2 \\
\hline & Somewhat unlikely & 4.3 & A minority & 20.1 \\
\hline & Extremely unlikely & 2.8 & Almost none & 4.3 \\
\hline \multirow{5}{*}{$\begin{array}{l}\text { Comply with a policy that requires me to } \\
\text { be tested for COVID-19 every week, } \\
\text { assuming that the university would } \\
\text { make tests available free of charge to } \\
\text { students. }\end{array}$} & Extremely likely & 63.6 & Almost all & 9.1 \\
\hline & Somewhat likely & 20.1 & A majority & 27.1 \\
\hline & $\begin{array}{l}\text { Neither likely nor } \\
\text { unlikely }\end{array}$ & 5.3 & About half & 35.2 \\
\hline & Somewhat likely & 5.8 & A minority & 24.9 \\
\hline & Extremely likely & 5.3 & Almost none & 3.8 \\
\hline \multirow{5}{*}{$\begin{array}{l}\text { Expect to be willing to comply with } \\
\text { updated health and safety protocols } \\
\text { being put in place. }\end{array}$} & Extremely likely & 76.4 & Almost all & 9.1 \\
\hline & Somewhat likely & 17.6 & A majority & 33.2 \\
\hline & $\begin{array}{l}\text { Neither likely nor } \\
\text { unlikely }\end{array}$ & 4.3 & About half & 39.2 \\
\hline & Somewhat unlikely & 0.5 & A minority & 14.6 \\
\hline & Extremely unlikely & 1.3 & Almost none & 4.0 \\
\hline \multirow{5}{*}{$\begin{array}{l}\text { Stay home from class if you had a fever } \\
\text { or other concerning symptoms }\end{array}$} & Extremely likely & 69.4 & Almost all & 17.1 \\
\hline & Somewhat likely & 19.4 & A majority & 35.4 \\
\hline & $\begin{array}{l}\text { Neither likely nor } \\
\text { unlikely }\end{array}$ & 5.8 & About half & 25.1 \\
\hline & Somewhat unlikely & 4.5 & A minority & 15.1 \\
\hline & Extremely unlikely & 1.0 & Almost none & 7.3 \\
\hline \multirow{5}{*}{$\begin{array}{l}\text { Comply with a policy that limit social } \\
\text { gatherings to small groups (no more } \\
\text { than } 20 \text { people) }\end{array}$} & Extremely likely & 49.0 & Almost all & 4.8 \\
\hline & Somewhat likely & 25.3 & A majority & 13.3 \\
\hline & $\begin{array}{l}\text { Neither likely nor } \\
\text { unlikely }\end{array}$ & 7.0 & About half & 32.7 \\
\hline & Somewhat unlikely & 12.0 & A minority & 37.7 \\
\hline & Extremely unlikely & 6.5 & Almost none & 11.6 \\
\hline \multirow{5}{*}{$\begin{array}{l}\text { Maintain } 6 \text { feet of social distance at } \\
\text { social gatherings (regardless of size) }\end{array}$} & Extremely likely & 23.1 & Almost all & 3.27 \\
\hline & Somewhat likely & 31.2 & A majority & 7.04 \\
\hline & $\begin{array}{l}\text { Neither likely nor } \\
\text { unlikely }\end{array}$ & 13.3 & About half & 21.4 \\
\hline & Somewhat unlikely & 19.9 & A minority & 41.7 \\
\hline & Extremely unlikely & 12.6 & Almost none & 26.6 \\
\hline
\end{tabular}


Table 4: Students' stated choices, current concern level, risk perception, and suitability of residence for online learning.

\begin{tabular}{|c|c|}
\hline Choices & $\%$ \\
\hline $\begin{array}{l}\text { Choice of lecture-based courses }(\mathrm{N}=398) \text { : } \\
\text { In-person } \\
\text { Exclusively online }\end{array}$ & $\begin{array}{l}46.2 \\
53.7\end{array}$ \\
\hline $\begin{array}{l}\text { Choice of lab-based courses }(\mathrm{N}=398) \text { : } \\
\text { In-person } \\
\text { Exclusively online }\end{array}$ & $\begin{array}{l}59.8 \\
40.2\end{array}$ \\
\hline $\begin{array}{l}\text { Choice with } \mathbf{5 0 \%} \text { Chance of Outbreak during Semester }(\mathrm{N}=396) \text { : } \\
\text { Start the semester with on-campus classes and move online if needed } \\
\text { Deliver all courses online for the Fall } 2020 \text { semester }\end{array}$ & $\begin{array}{l}54.5 \\
45.5\end{array}$ \\
\hline $\begin{array}{l}\text { Choice with } \mathbf{1 0 0 \%} \text { Chance of Outbreak during Semester ( } N=397) \text { : } \\
\text { Start the semester with on-campus classes and move online if needed } \\
\text { Deliver all courses online for the Fall } 2020 \text { semester }\end{array}$ & $\begin{array}{l}35.5 \\
64.5\end{array}$ \\
\hline $\begin{array}{l}\text { Current Concern Level }(\mathrm{N}=398) \text { : } \\
\text { Extremely concerned } \\
\text { Very concerned } \\
\text { Moderately concerned } \\
\text { Slightly concerned } \\
\text { Not at all concerned }\end{array}$ & $\begin{array}{l}21.6 \\
34.7 \\
29.6 \\
10.3 \\
3.5\end{array}$ \\
\hline $\begin{array}{l}\text { Perceived Risk of Infection ( } \mathrm{N}=396) \text { : } \\
\text { Very high } \\
\text { High } \\
\text { Moderate } \\
\text { Low } \\
\text { Very low } \\
\text { I don't understand the question }\end{array}$ & $\begin{array}{l}25.0 \\
23.2 \\
40.4 \\
9.1 \\
2.0 \\
0.3\end{array}$ \\
\hline $\begin{array}{l}\text { Perceived Risk of Adverse Effects if Infected ( } N=397) \text { : } \\
\text { Very high } \\
\text { High } \\
\text { Moderate } \\
\text { Low } \\
\text { Very low } \\
\text { I don't understand the question }\end{array}$ & $\begin{array}{l}8.8 \\
13.6 \\
27.0 \\
31.5 \\
16.9 \\
2.3\end{array}$ \\
\hline $\begin{array}{l}\text { Current Living Suitability for Online Courses ( } N=397) \text { : } \\
\text { Excellent } \\
\text { Good } \\
\text { Average } \\
\text { Poor } \\
\text { Terrible }\end{array}$ & $\begin{array}{l}32.7 \\
37.0 \\
22.4 \\
6.0 \\
1.8\end{array}$ \\
\hline
\end{tabular}


Table 5: Odds Ratios for Logistic Regression models of students' preferences for an in-person (1) course compared to an online (0) course

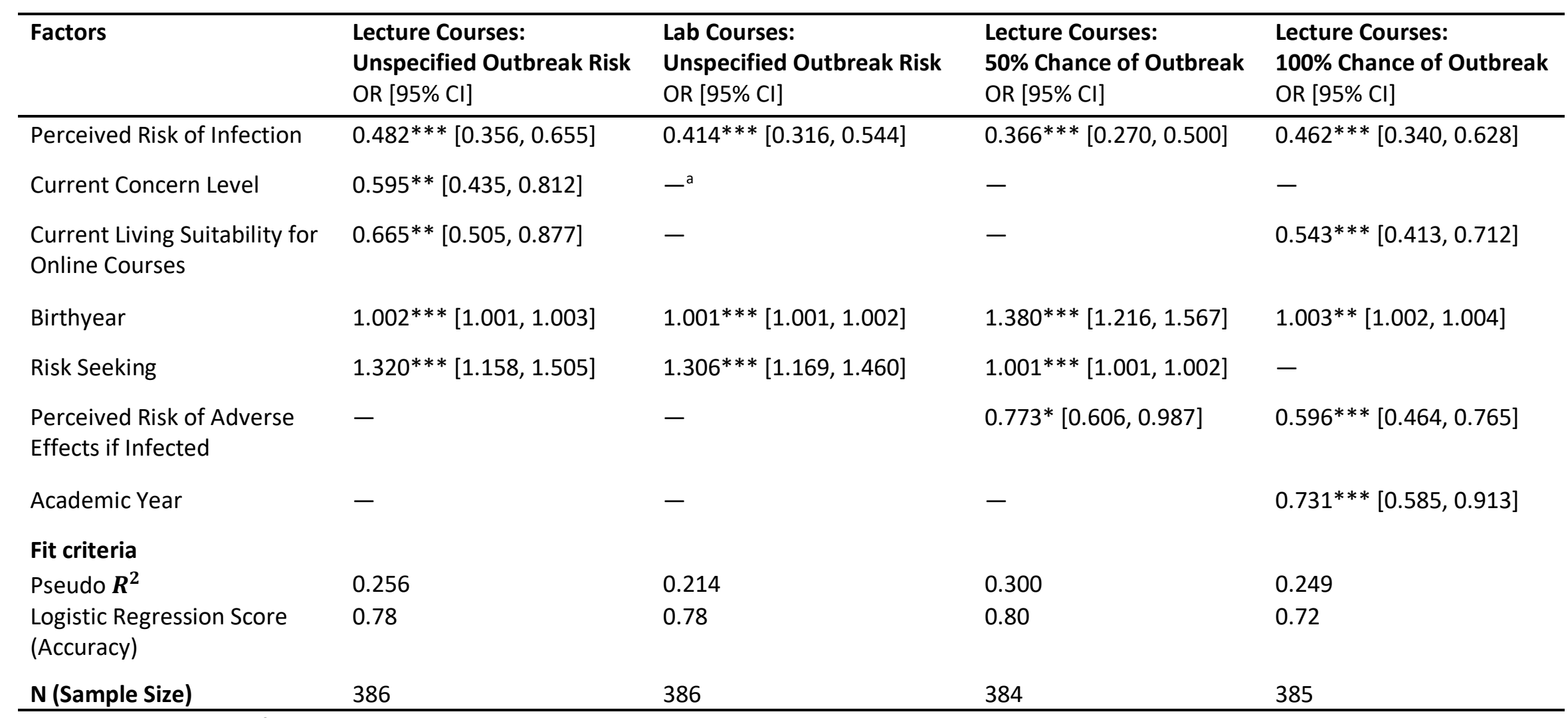

OR: odds ratio; $\mathrm{Cl}$ : confidence interval

${ }^{a}$ Dashes represent those factors that were not statistically significant and were accordingly removed during the recursive feature elimination process

${ }^{* * *} \boldsymbol{p}<0.001 ;{ }^{* *} \boldsymbol{p}<0.01 ;{ }^{*} \boldsymbol{p}<0.05$ 


\begin{tabular}{|c|c|c|c|c|c|c|}
\hline \multirow[t]{2}{*}{ Variables } & \multicolumn{6}{|c|}{ Coefficients and Standard Errors } \\
\hline & \multicolumn{2}{|c|}{$\begin{array}{l}\text { Class 1: } \\
\text { "Low-concern" } \\
\text { student }\end{array}$} & \multicolumn{2}{|c|}{$\begin{array}{l}\text { Class 2: } \\
\text { "Moderate- } \\
\text { concern" student }\end{array}$} & \multicolumn{2}{|c|}{$\begin{array}{l}\text { Class 3: } \\
\text { "High-concern" } \\
\text { student }\end{array}$} \\
\hline Class probability & 0.29 & & 0.54 & & 0.17 & \\
\hline Class membership model & Coef. & SE & Coef. & SE & Coef. & SE \\
\hline ASC & $-{ }^{a}$ & - & $-4.290 * * *$ & 0.901 & $-6.169 * * *$ & 1.367 \\
\hline Current concern level & - & - & $4.287 * * *$ & 0.898 & $4.468 * * *$ & 1.337 \\
\hline Choice of lecture-based courses & - & - & $3.724 * * *$ & 0.660 & $4.461 * * *$ & 0.733 \\
\hline Class-specific choice model & Coef. & SE & Coef. & SE & Coef. & SE \\
\hline ASC & $4.516^{* * *}$ & 0.664 & $2.959 * * *$ & 0.566 & -1.555 & 1.154 \\
\hline \multicolumn{7}{|l|}{ Mode of Course Delivery: } \\
\hline Entirely in-person ${ }^{b}$ & - & - & - & - & - & - \\
\hline Large online \& small in-person & $-0.514 * *$ & 0.198 & $1.967^{* * *}$ & 0.175 & $0.792 * *$ & 0.323 \\
\hline Some in-person \& some online & -0.219 & 0.191 & $1.478 * * *$ & 0.167 & 0.382 & 0.318 \\
\hline Entirely online & $-1.427^{* * *}$ & 0.393 & $2.619 * * *$ & 0.293 & $4.261 * * *$ & 0.728 \\
\hline \multicolumn{7}{|l|}{ Safety on Campus: } \\
\hline Required \& extensive & 0.340 & 0.272 & $2.126 * * *$ & 0.218 & $3.928 * * *$ & 0.633 \\
\hline Required \& some & $0.467 *$ & 0.232 & $1.859 * * *$ & 0.207 & $3.666 * * *$ & 0.632 \\
\hline Recommended \& some & $0.654 * * *$ & 0.185 & $1.541 * * *$ & 0.174 & $2.455 * * *$ & 0.583 \\
\hline No \& no ${ }^{b}$ & - & - & - & - & - & - \\
\hline \multicolumn{7}{|l|}{ Residence Hall Operating Capacity: } \\
\hline $0 \%$ & $-1.308 * * *$ & 0.267 & 0.342 & 0.242 & 0.064 & 0.414 \\
\hline $25 \%$ & $-0.484^{*}$ & 0.247 & $0.682 * * *$ & 0.204 & 0.175 & 0.421 \\
\hline $50 \%$ & -0.164 & 0.208 & 0.270 & 0.201 & -0.225 & 0.448 \\
\hline $100 \%^{b}$ & - & - & - & - & - & - \\
\hline Tuition Paid & $-0.013^{*}$ & 0.006 & $-0.049 * * *$ & 0.005 & $-0.030 * *$ & 0.01 \\
\hline \multirow{2}{*}{\multicolumn{7}{|c|}{$\begin{array}{l}\text { Limits on Events and Social } \\
\text { Gatherings: }\end{array}$}} \\
\hline & & & & & & \\
\hline 20 & $-0.967 * * *$ & 0.258 & $0.733 * * *$ & 0.201 & -0.350 & 0.396 \\
\hline 50 & -0.403 & 0.224 & $0.477^{*}$ & 0.198 & -0.641 & 0.397 \\
\hline 100 & 0.166 & 0.207 & $0.616 * * *$ & 0.188 & -0.768 & 0.409 \\
\hline No limit ${ }^{b}$ & - & - & - & - & - & - \\
\hline
\end{tabular}

Fit criteria

$\mathrm{AIC}=4616.70 ; \mathrm{BIC}=4906.39 ; \rho^{2}=0.3337$ (equally-likely base); Adjusted $\rho^{2}=0.3196$ (equally-likely base)

SE: standard error; ASC: alternative specific constant

${ }^{a}$ Coefficients for Class 1 in the class membership model are fixed at zero since it is the reference class

${ }^{\mathrm{b}}$ Coefficients of this level in the choice models are fixed at zero since it is the reference level

*** $p<0.001$; ** $p<0.01$; $^{*} p<0.05$ 
Table 7: Tuition percentage point equivalent (TPPE) of 3 latent classes $(N=386)$

\begin{tabular}{llll}
\hline Attributes and Levels & TPPE & & \\
\cline { 2 - 4 } & $\begin{array}{l}\text { Class 1: } \\
\text { "Low-concern" } \\
\text { student }\end{array}$ & $\begin{array}{l}\text { Class 2: } \\
\text { "Moderate- } \\
\text { concern" student }\end{array}$ & $\begin{array}{l}\text { Class 3: } \\
\text { "High-concern" } \\
\text { student }\end{array}$ \\
\hline ASC & $345.54 \%$ & $60.73 \%$ & $-52.61 \%$ \\
Mode of Course Delivery: & & & \\
$\quad$ Large online \& small in-person & $-39.34 \%$ & $40.36 \%$ & $26.79 \%$ \\
$\quad$ Some in-person \& some online & $-16.76 \%$ & $30.34 \%$ & $12.94 \%$ \\
$\quad$ Entirely online & $-109.16 \%$ & $53.74 \%$ & $144.20 \%$ \\
Safety on Campus: & & & \\
$\quad$ Required \& extensive & $26.04 \%$ & $43.63 \%$ & $132.92 \%$ \\
$\quad$ Required \& some & $35.77 \%$ & $38.15 \%$ & $124.06 \%$ \\
Recommended \& some & $50.05 \%$ & $31.61 \%$ & $83.07 \%$ \\
Residence Hall Operating Capacity: & & & \\
$\quad 0 \%$ & $-100.08 \%$ & $7.02 \%$ & $2.15 \%$ \\
$25 \%$ & $-37.00 \%$ & $14.00 \%$ & $-7.61 \%$ \\
$50 \%$ & $-12.57 \%$ & $5.55 \%$ & $-11.86 \%$ \\
Limits on Events and Social Gatherings: & & & $-21.70 \%$ \\
20 & $-74.01 \%$ & $15.05 \%$ & $-25.99 \%$ \\
50 & $-30.86 \%$ & $9.79 \%$ & \\
100 & $12.71 \%$ & $12.64 \%$ & \\
\hline
\end{tabular}

TPPE: Tuition percentage point equivalent; ASC: alternative specific constant 


\begin{tabular}{|c|c|c|c|}
\hline Variables & $\begin{array}{l}\text { Class 1: } \\
\text { "Low- } \\
\text { concern" } \\
\text { student }\end{array}$ & $\begin{array}{l}\text { Class 2: } \\
\text { "Moderate } \\
\text {-concern" } \\
\text { student }\end{array}$ & $\begin{array}{l}\text { Class 3: } \\
\text { "High- } \\
\text { concern" } \\
\text { student }\end{array}$ \\
\hline \multicolumn{4}{|l|}{ Stated Concern: } \\
\hline Current concern level ${ }^{a}$ & 2.78 & 3.93 & 4.02 \\
\hline Current concern versus concern in mid-March & 2.52 & 3.59 & 3.69 \\
\hline Risk seeking & 6.53 & 4.56 & 4.29 \\
\hline \multicolumn{4}{|l|}{ Student's Self-Reported Intentions: } \\
\hline Mask required (self) & 4.70 & 4.86 & 4.86 \\
\hline Mask recommended (self) & 4.08 & 4.60 & 4.60 \\
\hline Testing (self) & 4.11 & 4.42 & 4.40 \\
\hline Safety protocol (self) & 4.52 & 4.74 & 4.73 \\
\hline Stay home (self) & 4.62 & 4.47 & 4.45 \\
\hline Limit size of gatherings (self) & 3.50 & 4.18 & 4.22 \\
\hline Social distancing (self) & 2.88 & 3.49 & 3.54 \\
\hline \multicolumn{4}{|l|}{ Expectations for Classmates' Compliance Behavior: } \\
\hline Mask required (classmate) & 4.27 & 3.95 & 3.89 \\
\hline Mask recommended (classmate) & 3.35 & 3.13 & 3.08 \\
\hline Testing (classmate) & 3.31 & 3.08 & 3.03 \\
\hline Safety protocol (classmate) & 3.55 & 3.21 & 3.14 \\
\hline Stay home (classmate) & 3.79 & 3.27 & 3.19 \\
\hline Limit size of gatherings (classmate) & 2.73 & 2.59 & 2.58 \\
\hline Social distancing (classmate) & 2.28 & 2.15 & 2.13 \\
\hline \multicolumn{4}{|l|}{ Choice of Course's Delivery Mode: (online $=1$, in-person $=0$ ) } \\
\hline Choice of lecture-based courses ${ }^{\text {a }}$ & 0.07 & 0.70 & 0.83 \\
\hline Choice of lab-based courses & 0.16 & 0.49 & 0.54 \\
\hline \multicolumn{4}{|l|}{ Living Situation in Fall 2020: } \\
\hline Current Living Suitability for Online Courses & 3.57 & 4.05 & 4.11 \\
\hline Residential location in Fall 2020 (on campus $=1$, off campus $=0$ ) & 0.56 & 0.42 & 0.40 \\
\hline \multicolumn{4}{|l|}{ Perceived Risks: } \\
\hline Perceived risk of infection & 3.03 & 3.82 & 3.94 \\
\hline Perceived risk of adverse effects if infected & 2.05 & 2.88 & 2.98 \\
\hline \multicolumn{4}{|l|}{ Demographic Information: } \\
\hline Gender (female) & 0.52 & 0.51 & 0.50 \\
\hline Race (Asian / Pacific Islander) & 0.25 & 0.43 & 0.45 \\
\hline Race (Black / African American) & 0.01 & 0.03 & 0.03 \\
\hline Race (Hispanic / Latino) & 0.10 & 0.12 & 0.12 \\
\hline Race (White / Caucasian) & 0.64 & 0.43 & 0.40 \\
\hline Domestic students (yes $=1$, no $=0$ ) & 0.91 & 0.83 & 0.82 \\
\hline Political leaning (Democrat) & 0.37 & 0.56 & 0.58 \\
\hline Political leaning (Republican) & 0.25 & 0.14 & 0.12 \\
\hline Political leaning (Independent) & 0.33 & 0.27 & 0.26 \\
\hline World view (Conservative) & 0.20 & 0.09 & 0.09 \\
\hline World view (Moderate) & 0.51 & 0.49 & 0.49 \\
\hline World view (Liberal) & 0.29 & 0.41 & 0.42 \\
\hline
\end{tabular}

${ }^{a}$ Variables included in the class membership model 
Among the following options, which one do you prefer?

\begin{tabular}{|c|c|c|c|}
\hline & Enroll in Option 1 & Enroll in Option 2 & Option 3 (Defer) \\
\hline $\begin{array}{c}\text { Mode of course } \\
\text { delivery }\end{array}$ & All classes entirely online & $\begin{array}{l}\text { All classes deliver large } \\
\text { lectures online } \& \text { small } \\
\text { group activities in- } \\
\text { person }\end{array}$ & \multirow{5}{*}{$\begin{array}{l}\text { Defer enrollment for at } \\
\text { least one term }\end{array}$} \\
\hline Safety on Campus & $\begin{array}{c}\text { No masks \& no COVID- } \\
19 \text { testing }\end{array}$ & $\begin{array}{l}\text { Masks required \& some } \\
\text { COVID-19 testing }\end{array}$ & \\
\hline $\begin{array}{c}\text { Residence Hall } \\
\text { Operating Capacity }\end{array}$ & Closed, $0 \%$ capacity & Open, $100 \%$ capacity & \\
\hline Tuition Reduction & $10 \%$ & $30 \%$ & \\
\hline $\begin{array}{l}\text { Limits on Events and } \\
\text { Social Gatherings }\end{array}$ & No limit & 20 people & \\
\hline
\end{tabular}

\begin{tabular}{|l|c|c|c|}
\hline & Enroll in Option 1 & Enroll in Option 2 & $\begin{array}{c}\text { Defer Enrollment (Option } \\
\text { 3) }\end{array}$ \\
\hline Your choice: & 0 & 0 & 0 \\
\hline
\end{tabular}

Figure A1: Sample discrete choice experiment question. 


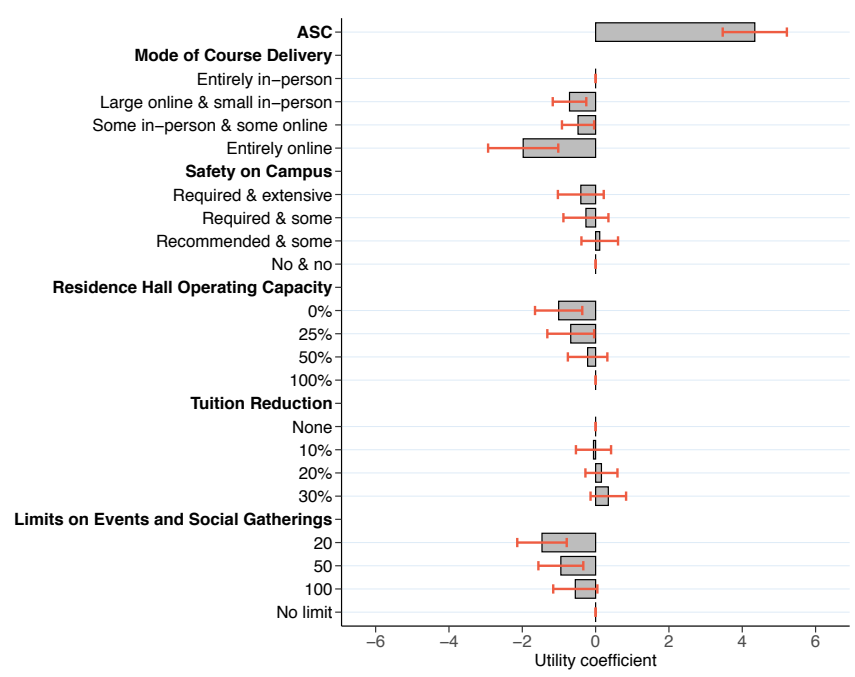

(a) "Not at all concerned" and "Slightly concerned" ( $N=52)$

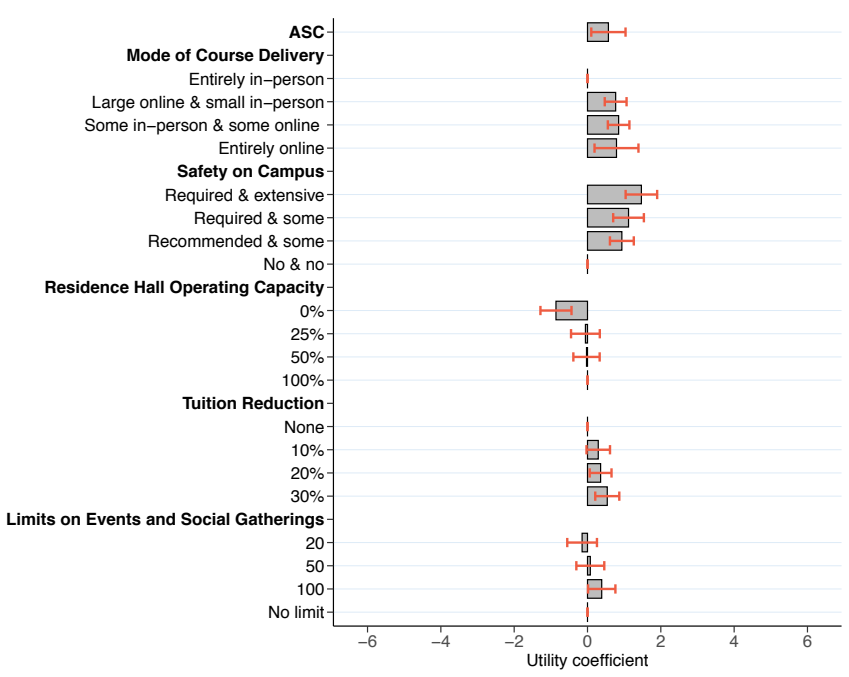

(b) "Moderately concerned" ( $N=118)$

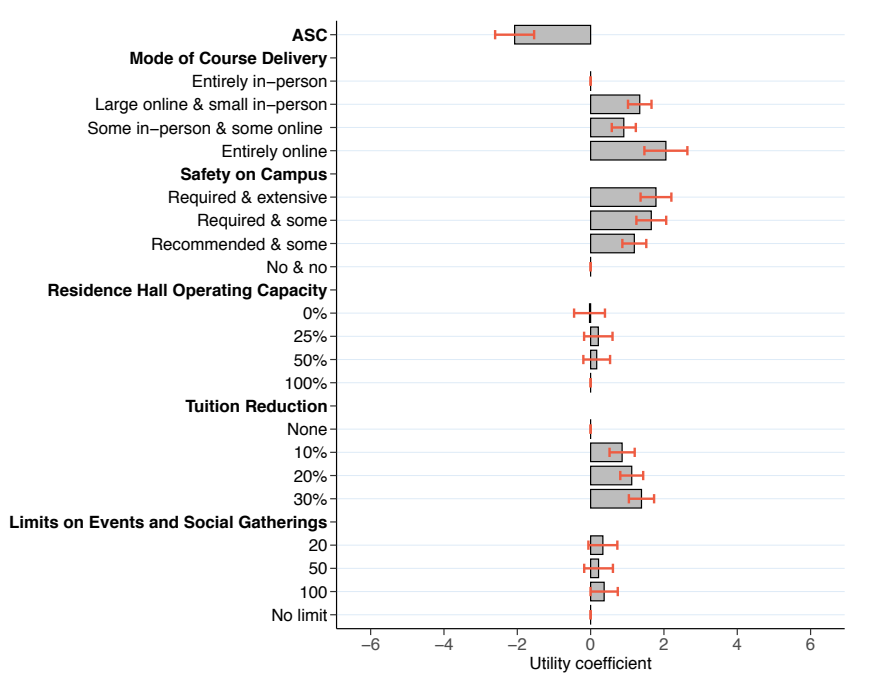

(c) "Very concerned" ( $N=133)$

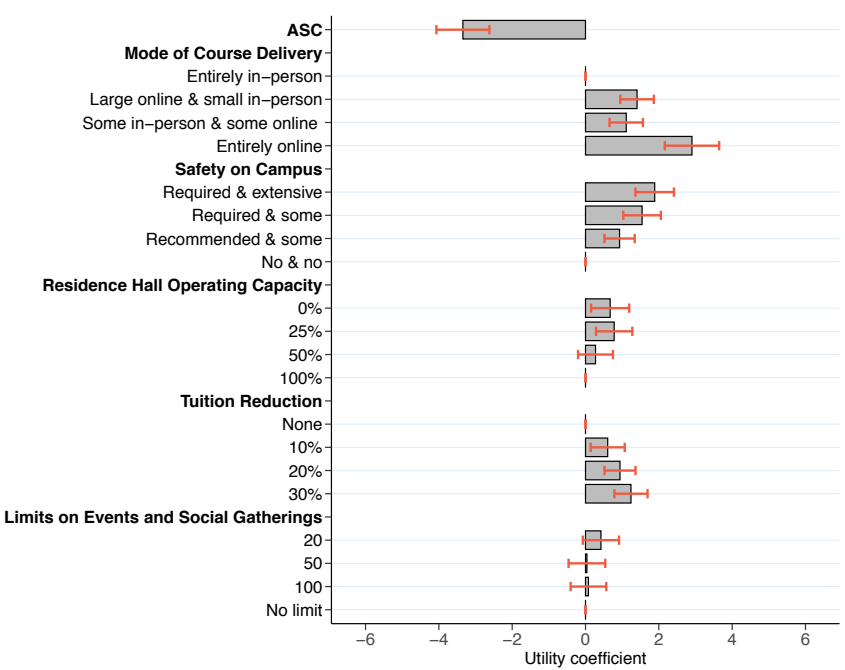

(d) "Extremely concerned" ( $N=83)$

Figure A2: Estimated results of conditional logit model for subgroup analysis of current concern level; (a) "Not at all concerned" and "Slightly concerned" ( $N=52)$; (b) "Moderately concerned" ( $N=118)$; (c) "Very concerned" ( $N=133)$; (d) "Extremely concerned" $(N=83)$ 


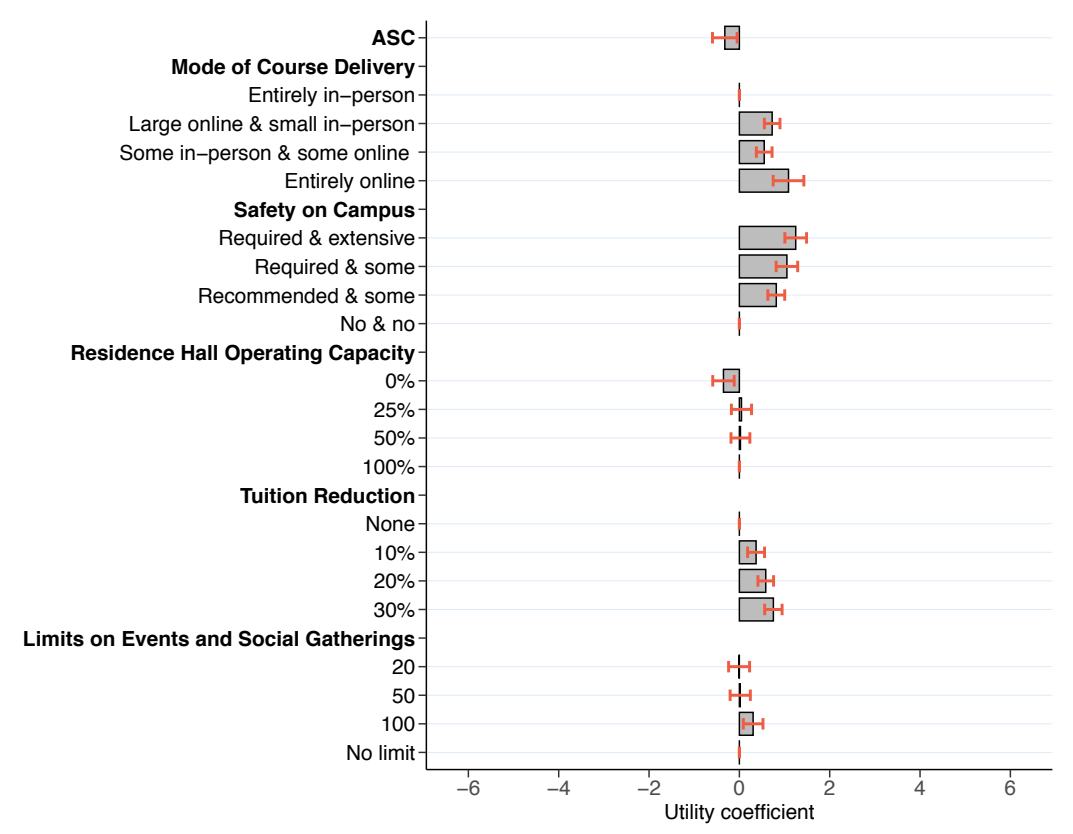

(a) "Domestic student" ( $N=329)$

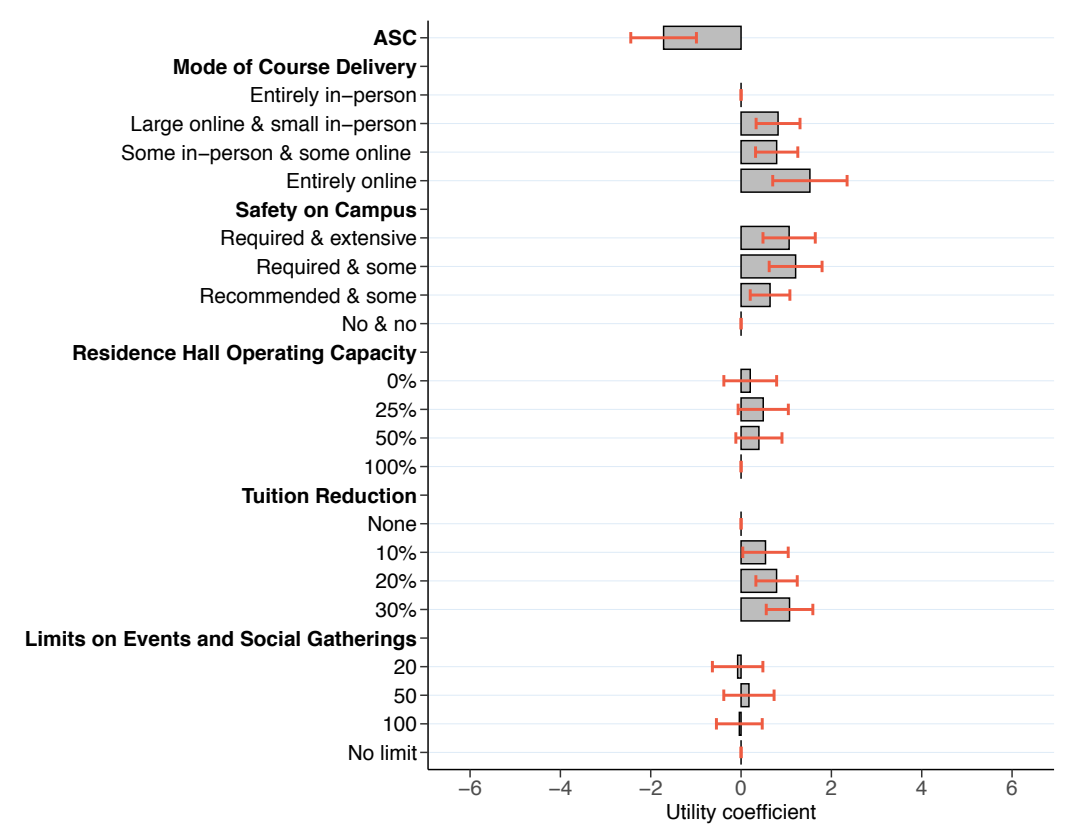

(b) "International student" ( $N=56)$

Figure A3: Estimated results of conditional logit model for subgroup analysis of domestic/international student; (a) "Domestic student" ( $N=329)$; (b) "International student" ( $N=56)$ 


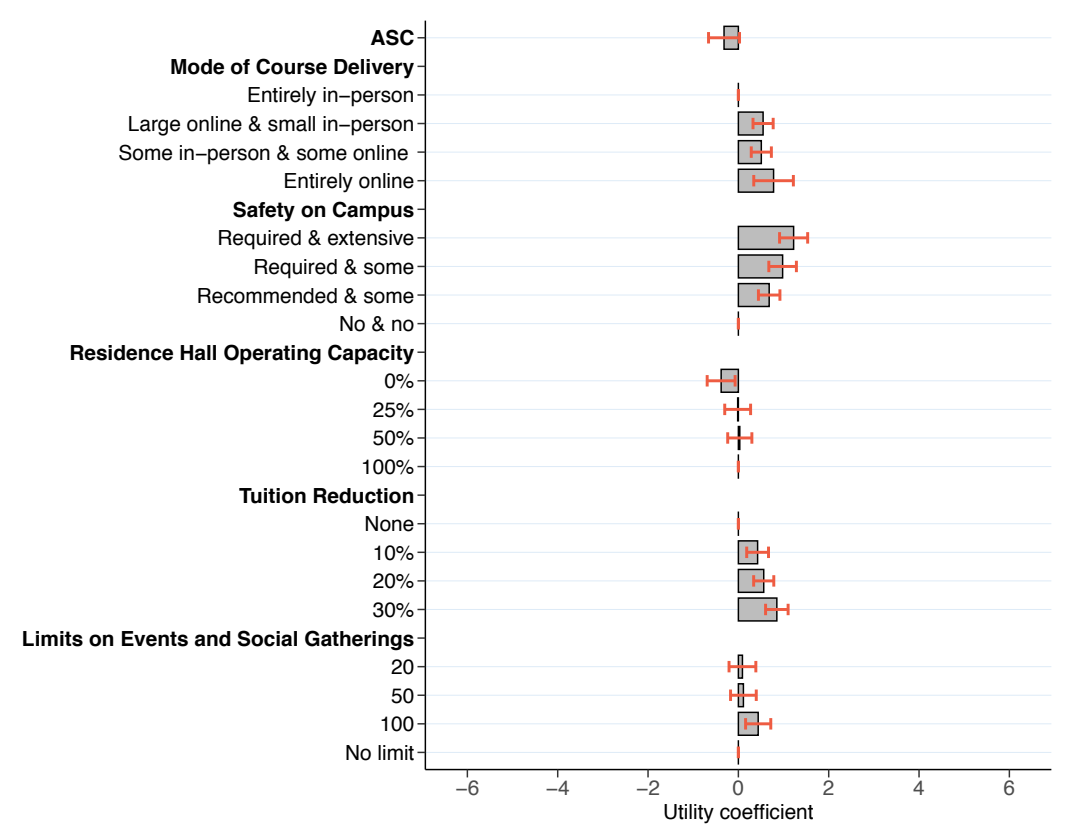

(a) "Female" ( $N=196)$

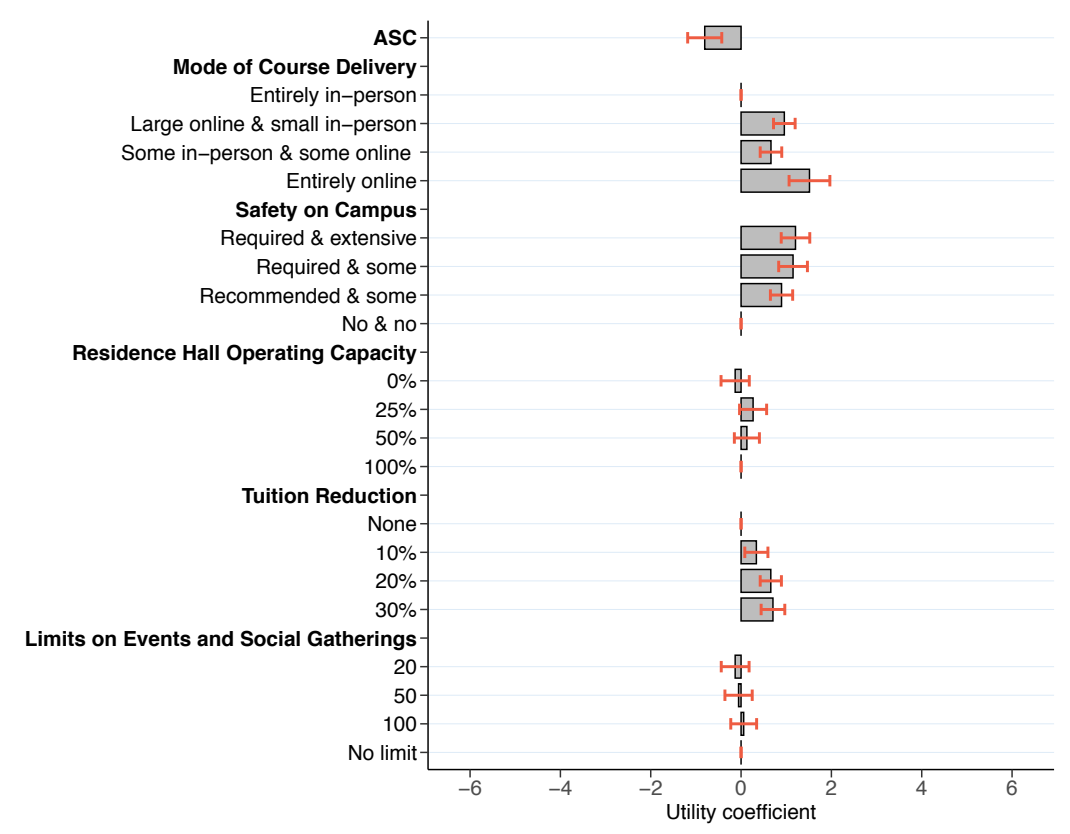

(b) "Male" ( $N=188)$

Figure A4: Estimated results of conditional logit model for subgroup analysis of gender; (a) "Female" ( $N=196)$; (b) "Male" (N=188) 


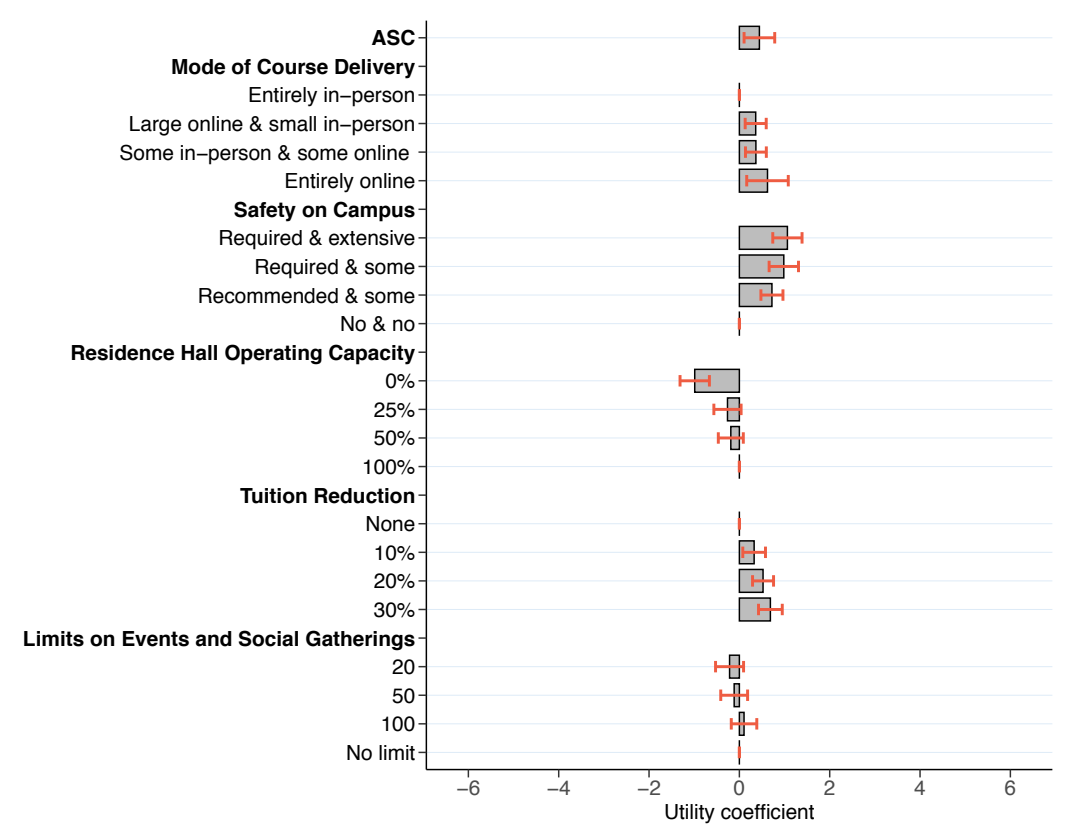

(a) "Living on campus" ( $N=176)$

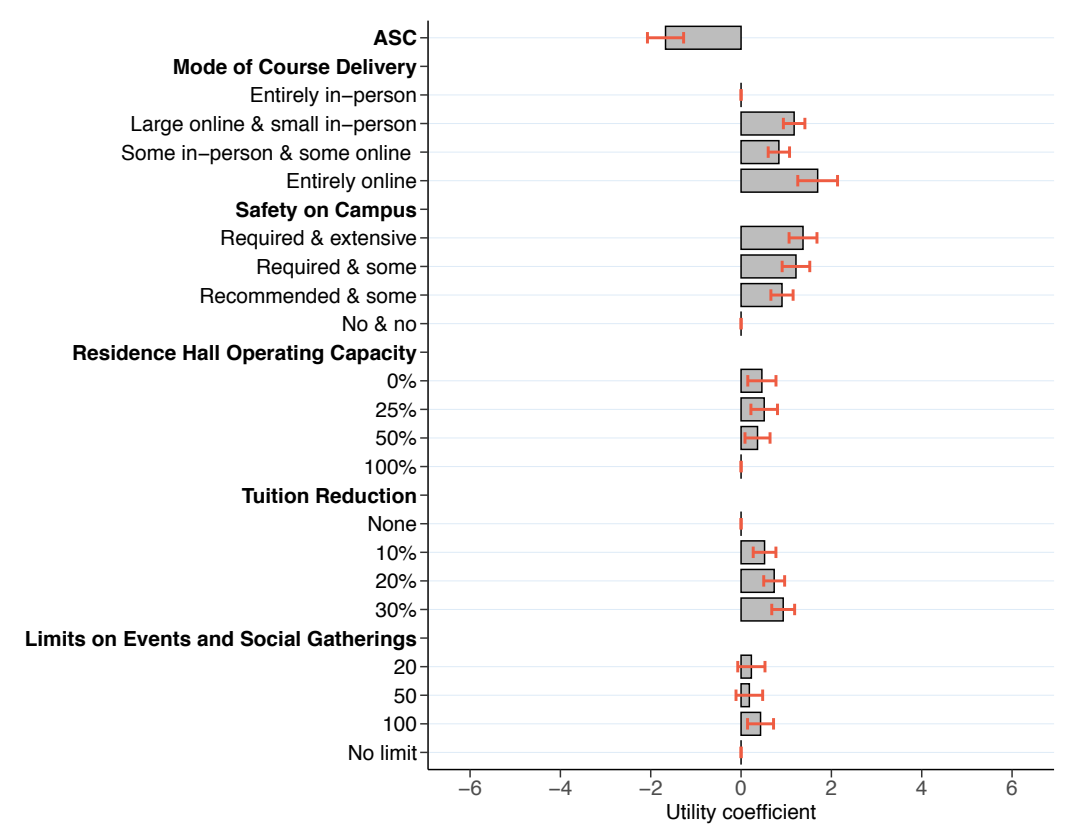

(b) "Living off campus" ( $N=210)$

Figure A5: Estimated results of conditional logit model for subgroup analysis of living location in Fall 2020; (a) "Living on campus" (N = 176); (b) "Living off campus" ( $N=210)$ 


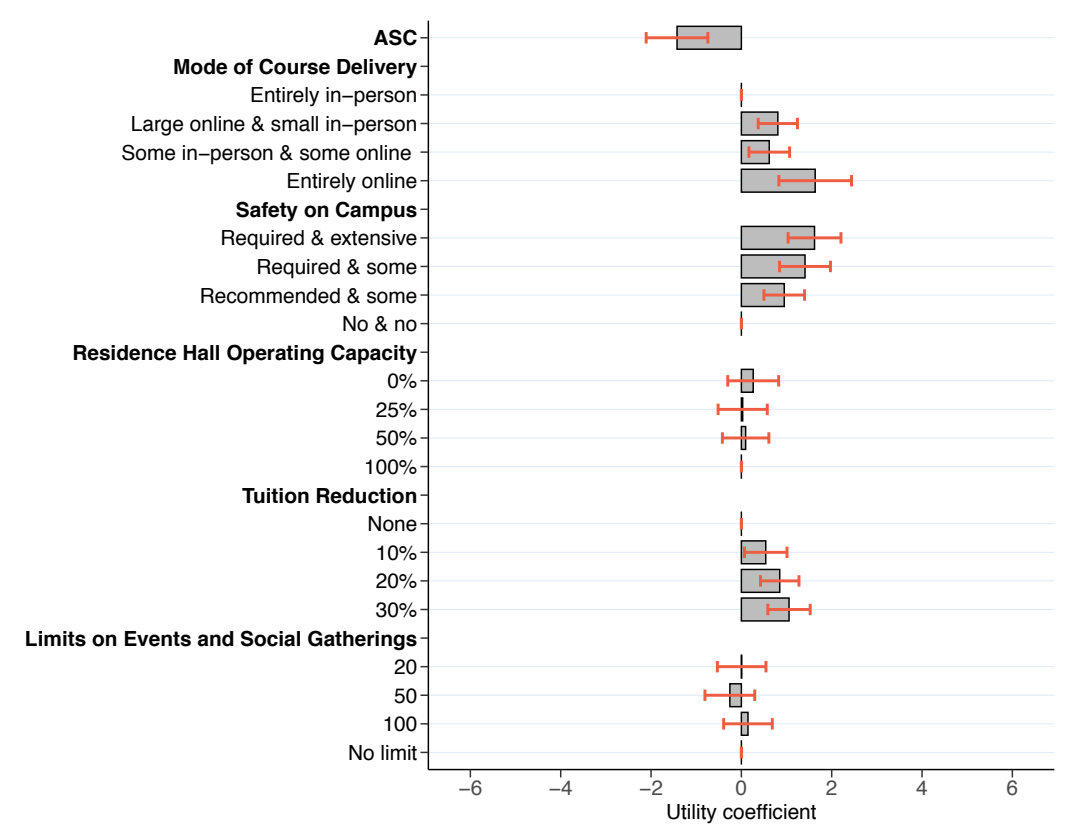

(a) "Yes" $(N=62)$

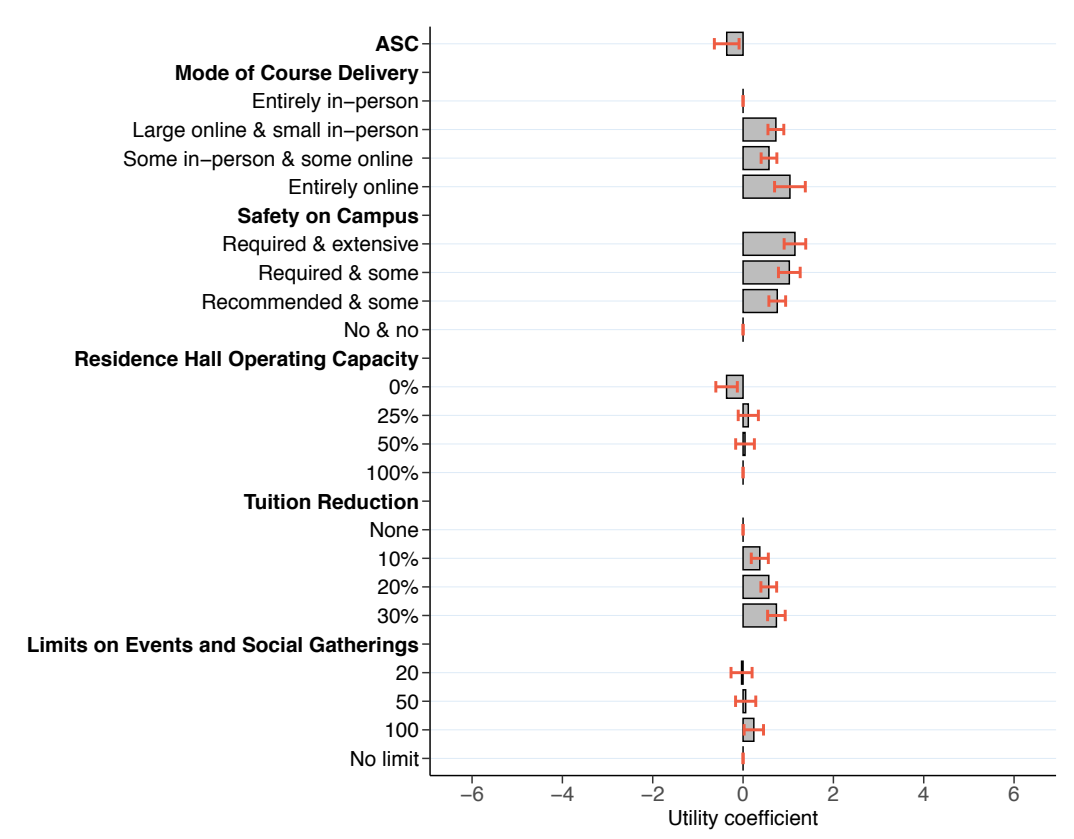

(b) “No" $(N=324)$

Figure A6: Estimated results of conditional logit model for subgroup analysis of having preexisting conditions or not; (a) "Yes" ( $N$ = 62); (b) "No" $(N=324)$ 


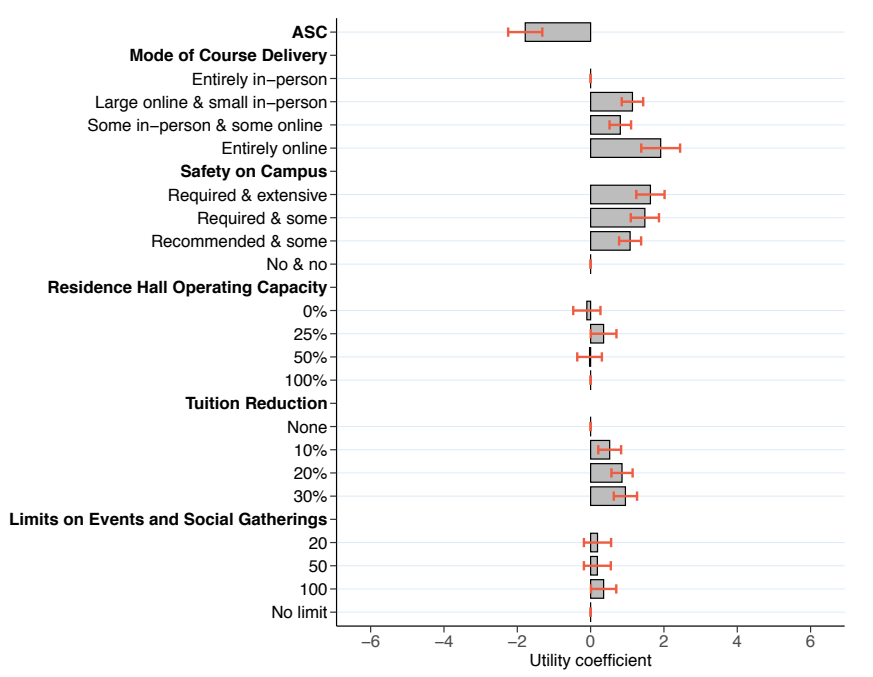

(a) "Asian / Pacific Islander" ( $N=142)$

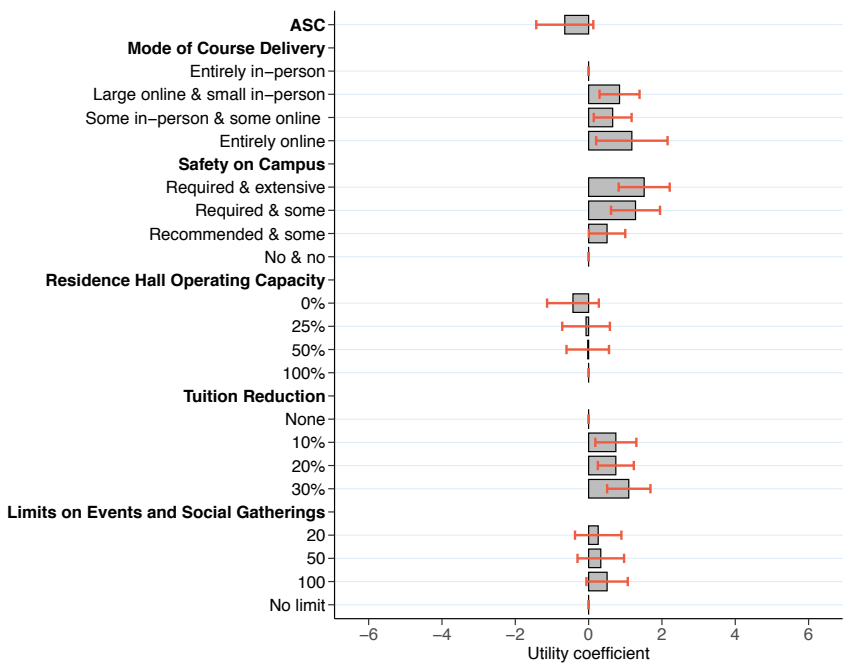

(c) "Hispanic / Latino" ( $\mathrm{N}=44)$

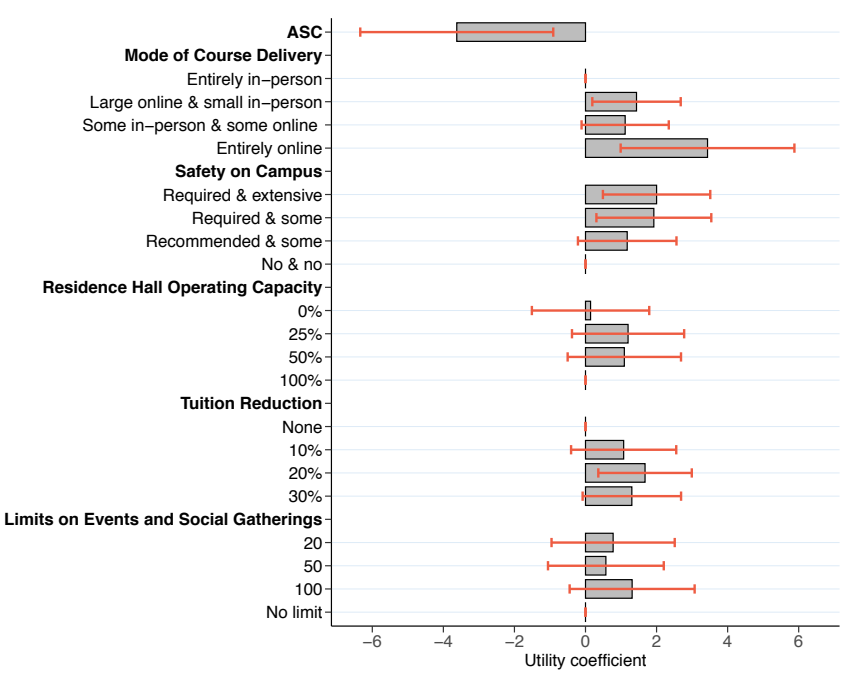

(b) "Black / African American" ( $N=10)$

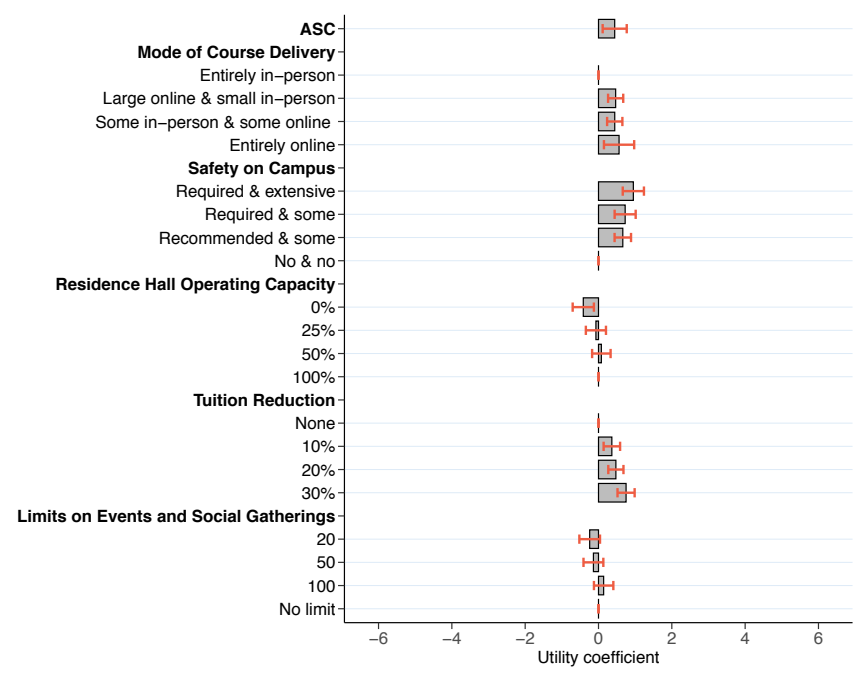

(d) "White / Caucasian" ( $N=215)$

Figure A7: Estimated results of conditional logit model for subgroup analysis of race; (a) "Asian / Pacific Islander" (N = 142); (b) "Black / African American" ( $N=10)$; (c) "Hispanic / Latino" ( $N=44)$; (d) "White / Caucasian" ( $N=215)$ 


\begin{tabular}{|c|c|c|c|}
\hline Variables & Coef. & SE & $95 \% \mathrm{Cl}$ \\
\hline ASC & $-0.524 * * *$ & 0.129 & {$[-0.777,-0.271]$} \\
\hline \multicolumn{4}{|l|}{ Mode of Course Delivery: } \\
\hline Entirely in-person ${ }^{a}$ & - & - & - \\
\hline Large online \& small in-person & $0.724 * * *$ & 0.082 & {$[0.562,0.885]$} \\
\hline Some in-person \& some online & $0.560 * * *$ & 0.082 & {$[0.399,0.721]$} \\
\hline Entirely online & $1.116 * * *$ & 0.159 & {$[0.804,0.427]$} \\
\hline \multicolumn{4}{|l|}{ Safety on Campus: } \\
\hline Required \& extensive & $1.209 * * *$ & 0.112 & {$[0.990,1.429]$} \\
\hline Required \& some & $1.074 * * *$ & 0.112 & {$[0.855,1.294]$} \\
\hline Recommended \& some & $0.786 * * *$ & 0.087 & {$[0.616,0.956]$} \\
\hline No \& no a & - & - & - \\
\hline \multicolumn{4}{|l|}{ Residence Hall Operating Capacity: } \\
\hline $0 \%$ & $-0.265^{*}$ & 0.111 & {$[-0.483,-0.047]$} \\
\hline $25 \%$ & 0.108 & 0.105 & {$[-0.098,0.313]$} \\
\hline $50 \%$ & 0.065 & 0.098 & {$[-0.127,0.256]$} \\
\hline $100 \%^{a}$ & - & - & - \\
\hline \multicolumn{4}{|l|}{ Tuition Reduction: } \\
\hline None ${ }^{a}$ & - & - & - \\
\hline $10 \%$ & $0.390 * * *$ & 0.089 & {$[0.215,0.564]$} \\
\hline $20 \%$ & $0.613 * * *$ & 0.082 & {$[0.453,0.773]$} \\
\hline $30 \%$ & $0.790 * * *$ & 0.091 & {$[0.611,0.969]$} \\
\hline \multicolumn{4}{|c|}{ Limits on Events and Social Gatherings: } \\
\hline 20 & -0.029 & 0.109 & {$[-0.242,0.184]$} \\
\hline 50 & 0.021 & 0.105 & {$[-0.186,0.227]$} \\
\hline 100 & $0.238^{*}$ & 0.101 & {$[0.040,0.436]$} \\
\hline No limit ${ }^{a}$ & - & - & - \\
\hline \multicolumn{4}{|l|}{ Fit criterion } \\
\hline \multicolumn{4}{|c|}{$\begin{array}{l}\text { AIC }=5688.32 ; \mathrm{BIC}=5784.89 ; \rho^{2}=0.1664 \text { (equally-likely base); Adjusted } \rho^{2}=0.1616 \text { (equally-likely } \\
\text { base) }\end{array}$} \\
\hline
\end{tabular}


Table A2: Fit criteria of latent class models by number of classes ( $N=386)$

\begin{tabular}{lllll}
\hline Number of classes & AIC & BIC & $\boldsymbol{\rho}^{2}$ (equally-likely base) & Adjusted $\boldsymbol{\rho}^{\mathbf{2}}$ (equally-likely base); \\
\hline 2 & 4873.89 & 5060.17 & 0.2719 & 0.2626 \\
3 & 4616.70 & 4906.39 & 0.3337 & 0.3196 \\
4 & 4455.23 & 4823.23 & 0.3597 & 0.3417 \\
\hline
\end{tabular}




\section{Appendix B: Design of Discrete Choice Experiment}

In this appendix, we detail our experimental design for the discrete choice survey. The following are the attributes and levels considered for the choice sets. The corresponding labels indicate which variable corresponds to this level in the first and second choices.

Attributes \& Levels

1-Mode of Course delivery: (4 levels) [“Mode"]

- All classes delivered entirely in-person; (level 1)*

- All classes deliver large lectures online \& small group activities in-person (level 2)

- Some classes delivered entirely in-person or some classes delivered entirely online (level 3)

- All classes delivered entirely online (level 4)

2 - Safety in Campus: (4 levels) ["Campus Safety"]

- Masks required \& extensive COVID-19 testing (level 1)

- Masks required \& some COVID-19 testing (level 2)

- Masks recommended \& some COVID-19 testing (level 3)

- No masks \& no testing (level 4)*

3 - Residence Hall Operating capacity (4 levels) [“Housing”]

- Closed, $0 \%$ (level 1)

- Open at $25 \%$ capacity (No roommates \& no shared bathrooms) (level 2)

- Open, $50 \%$ capacity (no roommates, but shared bathrooms) (level 3)

- Open, $100 \%$ capacity (level 4$)^{*}$

4-Tuition Reduction (4 levels) ["Tuition"]

- None (level 1)*

- $10 \%$ (level 2)

- $20 \%$ (level 3)

- $30 \%$ (level 4$)$

5 -Limits on events and social gatherings (4 levels) [“Campus Life”]

- No more than 20 people (level 1)

- No more than 50 people (level 2)

- No more than 100 people (level 3)

- No limit (level 4)* 
The following is a list of restrictions on the design in the choice set.

\section{List of restrictions on the design:}

1. If a choice includes "All courses delivered completely online", then the level for Campus Safety must be "No Masks \& No COVID-19 Testing", the level for Housing must be "Closed, 0\%", and the limits on social gatherings must be "No limit"

2. Do not allow options that are identical except for tuition, because one of the choices will be dominated.

3. If a choice requires masks, there must be limits on campus housing (cannot offer " $100 \%$ capacity) and limits on events (cannot offer "No Limit").

4. If a choice includes "All courses completely in-person", the tuition reduction for this option must be less than or equal to the tuition for the other option.

5. If a choice includes "All courses completely online, the tuition reduction for this option must be greater than or equal to the tuition for the other option.

6. For choice sets where neither option includes "All courses completely online", the choice sets must have a consistent ordering on the "Campus Safety", "Housing" and "Limits on Events" attributes in terms of their "strict" to "relaxed" policies. That is Option 1 must have all attributes at least as strict as Option 2 or vice versa. We remove choice sets with "All courses" completely online because of the extreme values on the other attributes.

- Campus safety: 1 (Most strict) to 4 (Most Relaxed)

- Housing: 1 (Most strict) to 4 (Most relaxed)

- Events: 1 (Most strict) to 4 (Most relaxed)

When only restrictions \#1 and \#2 are shown, the D-efficiency was 88\%. Under restrictions 1-6, the Defficiency was $75 \%$. 


\section{Appendix C: The Survey Instrument}

\section{[Section 0 - Basic Questions]}

0.1 Are you at least 18 years old?
a. Yes

b. No

0.2 Are you a citizen of the European Union? (Your answer will direct you to the appropriate consent forms).
a. Yes

b. No

The purpose of this survey is to better understand the impact of the COVID-19 (coronavirus) pandemic on student views related to the re-opening of colleges/universities in Fall 2020. Note that this survey is for research purposes and is not necessarily a reflection of the policies being considered at your college/university. However, summary statistics from this survey will be communicated to planners at colleges and universities. Your responses are anonymous, and we are not collecting any identifying information such as your IP address.

Please note that this survey is best taken on a device that has a screen the size of a tablet or larger.

\section{[Section 1 - Stated Concern]}

1.1 How concerned are you about the COVID-19/coronavirus pandemic currently?
a. Not at all concerned
b. Slightly concerned
c. Moderately concerned
d. Very concerned
e. Extremely concerned 
1.2 Around mid-March, many colleges/universities such as Stanford University, University of Michigan, Harvard University, and Georgia Institute of Technology announced that classes would be delivered online for the remainder of the semester due to COVID19 (the coronavirus). How does your level of current concern about the pandemic compare to your level of concern in mid-March?
a. I am currently much less concerned than I was in mid-March
b. I am currently a little less concerned than I was in mid-March
c. I have about the same level of concern
d. I am currently a little more concerned than I was in mid-March
e. I am currently much more concerned than I was in mid-March

1.3 In general, how willing are you to take risks? Please use a scale from 0 to 10, where a 0 means you are "completely unwilling to take risks" and a 10 means you are "very willing to take risks." Feel free to select a range of numbers if that best describes your willingness.

\begin{tabular}{l}
\begin{tabular}{l} 
Completely \\
Unwilling to \\
Take risks \\
\hline 0
\end{tabular}$\quad 1$ \\
\hline
\end{tabular}

1.4 Suppose you are facing a lottery with two possible outcomes, one with a low payoff and one with a high payoff. In every lottery, the low and high outcomes are equally likely to occur. In other words, each lottery is similar to flipping a fair coin. Which of the following six lotteries would you select?

\begin{tabular}{|l|l|l|l|l|l|}
\hline Lottery & Low Payoff & High Payoff & $\begin{array}{l}\text { Chance of Low } \\
\text { Payoff }\end{array}$ & $\begin{array}{l}\text { Chance of High } \\
\text { Payoff }\end{array}$ & $\begin{array}{l}\text { Your Selection } \\
\text { (mark only one) }\end{array}$ \\
\hline 1 & $\$ 28$ & $\$ 28$ & $50 \%$ & $50 \%$ & \\
\hline
\end{tabular}




\begin{tabular}{|l|l|l|l|l|l|}
\hline 2 & $\$ 24$ & $\$ 36$ & $50 \%$ & $50 \%$ & \\
\hline 3 & $\$ 20$ & $\$ 44$ & $50 \%$ & $50 \%$ & \\
\hline 4 & $\$ 16$ & $\$ 52$ & $50 \%$ & $50 \%$ & \\
\hline 5 & $\$ 12$ & $\$ 60$ & $50 \%$ & $50 \%$ & \\
\hline 6 & $\$ 2$ & $\$ 70$ & $50 \%$ & $50 \%$ & \\
\hline
\end{tabular}

\section{[Section 2 - Discrete Choice]}

Imagine a future August 2020 in which the COVID-19 (coronavirus) pandemic still presents an issue in the United States and worldwide. In the following questions, you will be presented with a series of choices about options for Fall 2020. For each group of options described, please select which one you would choose in this version of the future, assuming that you are taking all lecturebased courses (no lab-based classes).

You are going to be presented with 8 scenarios. After these 8 scenarios, the rest of the survey should be much more straightforward!

2.1 Among the following options, which one do you prefer? [Example question given here]

\begin{tabular}{|c|c|c|c|}
\hline & Enroll in Option 1 & Enroll in Option 2 & Option 3 (Defer) \\
\hline Mode of course delivery & $\begin{array}{l}\text { Some classes \& some } \\
\text { classes entirely in person }\end{array}$ & $\begin{array}{l}\text { All classes entirely in- } \\
\text { person }\end{array}$ & \multirow{3}{*}{$\begin{array}{l}\text { Defer enrollment } \\
\text { for at least one } \\
\text { term }\end{array}$} \\
\hline Safety on campus & $\begin{array}{l}\text { No masks \& no COVID-19 } \\
\text { testing }\end{array}$ & $\begin{array}{l}\text { Masks recommended } \\
\text { \& some COVID-19 } \\
\text { testing }\end{array}$ & \\
\hline Residence Hall Operating Capacity & $\begin{array}{l}\text { Open, } 25 \% \text { capacity (no } \\
\text { roommates \& no shared } \\
\text { bathrooms) }\end{array}$ & $\begin{array}{l}\text { Open, } 25 \% \text { capacity } \\
\text { (No roommates \& no } \\
\text { shared bathrooms) }\end{array}$ & \\
\hline
\end{tabular}




\begin{tabular}{|c|c|c|c|}
\hline Tuition Reduction & $30 \%$ & $20 \%$ & \\
\hline Limits on Events and Social Gatherings & 50 people & 20 people & \\
\hline & Enroll in Option 1 & Enroll in Option 2 & Defer (Option 3) \\
\hline Your choice: & & & \\
\hline
\end{tabular}

2.2 Among the following options, which one do you prefer?

\begin{tabular}{|c|c|c|c|}
\hline & Enroll in Option 1 & Enroll in Option 2 & Option 3 (Defer) \\
\hline Mode of course delivery & [Level of Attribute] & [Level of Attribute] & \multirow{5}{*}{$\begin{array}{l}\text { Defer enrollment } \\
\text { for at least one } \\
\text { term }\end{array}$} \\
\hline Safety on campus & [Level of Attribute] & [Level of Attribute] & \\
\hline Residence Hall Operating Capacity & [Level of Attribute] & [Level of Attribute] & \\
\hline Tuition Reduction & [Level of Attribute] & [Level of Attribute] & \\
\hline \multirow[t]{2}{*}{ Limits on Events and Social Gatherings } & [Level of Attribute] & [Level of Attribute] & \\
\hline & Enroll in Option 1 & Enroll in Option 2 & Defer (Option 3) \\
\hline Your choice: & & & \\
\hline
\end{tabular}

2.3 Among the following options, which one do you prefer?

\begin{tabular}{|l|l|l|l|}
\hline & Enroll in Option 1 & Enroll in Option 2 & Option 3 (Defer) \\
\hline Mode of course delivery & [Level of Attribute] & [Level of Attribute] & $\begin{array}{l}\text { Defer enrollment } \\
\text { for at least one } \\
\text { term }\end{array}$ \\
\hline Rafety on campus & [Level of Attribute] & [Level of Attribute] & [Level of Attribute]
\end{tabular}




\begin{tabular}{|l|l|l|l|}
\hline Tuition Reduction & [Level of Attribute] & [Level of Attribute] & \\
\hline Limits on Events and Social Gatherings & [Level of Attribute] & [Level of Attribute] & \\
\hline & \multicolumn{3}{|l|}{} \\
\hline Your choice: & Enroll in Option 1 & Enroll in Option 2 & Defer (Option 3) \\
\hline
\end{tabular}

2.4 Among the following options, which one do you prefer?

\begin{tabular}{|l|l|l|l|}
\hline & Enroll in Option 1 & Enroll in Option 2 & Option 3 (Defer) \\
\hline Mode of course delivery & [Level of Attribute] & [Level of Attribute] & $\begin{array}{l}\text { Defer enrollment } \\
\text { for at least one } \\
\text { term }\end{array}$ \\
\hline Safety on campus & [Level of Attribute] & [Level of Attribute] & [Level of Attribute] \\
\hline Residence Hall Operating Capacity & [Level of Attribute] & [Level of Attribute] \\
\hline Tuition Reduction & [Level of Attribute] & [Level of Attribute] & \\
\hline Limits on Events and Social Gatherings & [Level of Attribute] & Enroll in Option 2 & Defer (Option 3) \\
\hline & \multicolumn{2}{|l|}{} \\
\hline Your choice: & Enroll in Option 1 & \\
\hline
\end{tabular}

2.5 Among the following options, which one do you prefer?

\begin{tabular}{|l|l|l|l|}
\hline & Enroll in Option 1 & Enroll in Option 2 & Option 3 (Defer) \\
\hline Mode of course delivery & [Level of Attribute] & [Level of Attribute] & $\begin{array}{l}\text { Defer enrollment } \\
\text { for at least one } \\
\text { term }\end{array}$ \\
\hline Residence Hall Operating Capacity & [Level of Attribute] & [Level of Attribute] & [Level of Attribute] \\
\hline
\end{tabular}




\begin{tabular}{|l|l|l|l|}
\hline Tuition Reduction & [Level of Attribute] & [Level of Attribute] & \\
\hline Limits on Events and Social Gatherings & [Level of Attribute] & [Level of Attribute] & \\
\hline & \multicolumn{3}{|l|}{} \\
\hline Your choice: & Enroll in Option 1 & Enroll in Option 2 & Defer (Option 3) \\
\hline
\end{tabular}

2.6 Among the following options, which one do you prefer?

\begin{tabular}{|c|c|c|c|}
\hline & Enroll in Option 1 & Enroll in Option 2 & Option 3 (Defer) \\
\hline Mode of course delivery & [Level of Attribute] & [Level of Attribute] & \multirow{5}{*}{$\begin{array}{l}\text { Defer enrollment } \\
\text { for at least one } \\
\text { term }\end{array}$} \\
\hline Safety on campus & [Level of Attribute] & [Level of Attribute] & \\
\hline Residence Hall Operating Capacity & [Level of Attribute] & [Level of Attribute] & \\
\hline Tuition Reduction & [Level of Attribute] & [Level of Attribute] & \\
\hline \multirow[t]{2}{*}{ Limits on Events and Social Gatherings } & [Level of Attribute] & [Level of Attribute] & \\
\hline & Enroll in Option 1 & Enroll in Option 2 & Defer (Option 3) \\
\hline Your choice: & & & \\
\hline
\end{tabular}

2.7 Among the following options, which one do you prefer?

\begin{tabular}{|l|l|l|l|}
\hline & Enroll in Option 1 & Enroll in Option 2 & Option 3 (Defer) \\
\hline Mode of course delivery & [Level of Attribute] & [Level of Attribute] & $\begin{array}{l}\text { Defer enrollment } \\
\text { for at least one } \\
\text { term }\end{array}$ \\
\hline Rafety on campus & [Level of Attribute] & [Level of Attribute] & [Level of Attribute]
\end{tabular}




\begin{tabular}{|l|l|l|l|}
\hline Tuition Reduction & [Level of Attribute] & [Level of Attribute] & \\
\hline Limits on Events and Social Gatherings & [Level of Attribute] & [Level of Attribute] & \\
\hline & \multicolumn{3}{|l|}{} \\
\hline & Enroll in Option 1 & Enroll in Option 2 & Defer (Option 3) \\
\hline Your choice: & & & \\
\hline
\end{tabular}

2.8 Among the following options, which one do you prefer?

\begin{tabular}{|c|c|c|c|}
\hline & Enroll in Option 1 & Enroll in Option 2 & Option 3 (Defer) \\
\hline Mode of course delivery & [Level of Attribute] & [Level of Attribute] & \multirow{5}{*}{$\begin{array}{l}\text { Defer enrollment } \\
\text { for at least one } \\
\text { term }\end{array}$} \\
\hline Safety on campus & [Level of Attribute] & [Level of Attribute] & \\
\hline Residence Hall Operating Capacity & [Level of Attribute] & [Level of Attribute] & \\
\hline Tuition Reduction & [Level of Attribute] & [Level of Attribute] & \\
\hline \multirow[t]{2}{*}{ Limits on Events and Social Gatherings } & [Level of Attribute] & [Level of Attribute] & \\
\hline & Enroll in Option 1 & Enroll in Option 2 & Defer (Option 3) \\
\hline Your choice: & & & \\
\hline
\end{tabular}

\section{[Section 3 - Student's Self-Reported Intentions to Comply with Safety Protocols]}

Suppose your college/university has on-campus classes in Fall 2020. How likely are you to engage in the following practices?

3.1 I will wear a cloth face covering while moving around campus and in instructional space, if required.

a. Extremely likely 

b. Somewhat likely
c. Neither likely nor unlikely
d. Somewhat unlikely
e. Extremely unlikely

3.2 I will wear a cloth face covering while moving around campus and in instructional space, if recommended, but not required.
a. Extremely likely
b. Somewhat likely
c. Neither likely nor unlikely
d. Somewhat unlikely
e. Extremely unlikely

3.3 I will comply with a policy that requires me to be tested for COVID-19 every week, assuming that the university would make tests available free of charge to students.
a. Extremely likely
b. Somewhat likely
c. Neither likely nor unlikely
d. Somewhat unlikely
e. Extremely unlikely

3.4 I expect to be willing to comply with updated health and safety protocols being put in place.
a. Strongly agree
b. Agree
c. Neither agree nor disagree
d. Disagree
e. Strongly disagree 
3.5 How likely are you to stay home from class if you had a fever or other concerning symptoms?
a. Extremely likely
b. Somewhat likely
c. Neither likely nor unlikely
d. Somewhat unlikely
e. Extremely unlikely

3.6 How likely are you to limit the size of your social gatherings to small groups (no more than 20 people)?
a. Extremely likely
b. Somewhat likely
c. Neither likely nor unlikely
d. Somewhat unlikely
e. Extremely unlikely

3.7 How likely are you to maintain 6 feet of social distance at your social gatherings (regardless of size)?
a. Extremely likely
b. Somewhat likely
c. Neither likely nor unlikely
d. Somewhat unlikely
e. Extremely unlikely

\section{[Section 4 - Expectations for Classmates' Compliance Behavior]}

Suppose your college/university has on-campus classes in Fall 2020. What percentage of your classmates do you expect to engage in the following practices? 
4.1 How many of your classmates do you expect would wear a cloth face covering while moving around campus and in instructional space, if required?
a. Almost none
b. A minority
c. About half
d. A majority
e. Almost all

4.2 How many of your classmates do you expect would wear a cloth face covering while moving around campus and in instructional space, if recommended but not required?
a. Almost none
b. A minority
c. About half
d. A majority
e. Almost all

4.3 How many of your classmates do you expect would comply with a university policy that required students to get tested for COVID-19 every week, assuming that the university would make tests available free of charge to students?
a. Almost none
b. A minority
c. About half
d. A majority
e. Almost all

4.4 How many of your classmates do you expect to comply with updated health and safety protocols that are expected to be put in place?
a. Almost none
b. A minority 

c. About half
d. A majority
e. Almost all

4.5 How many of your classmates do you expect would stay home from class if they had a fever or other concerning symptoms?
a. Almost none
b. A minority
c. About half
d. A majority
e. Almost all

4.6 How many of your classmates do you expect would limit social gatherings to small groups (no more than 20 people)?
a. Almost none
b. A minority
c. About half
d. A majority
e. Almost all

4.7 How many of your classmates do you expect would maintain 6 feet of social distance at social gatherings (regardless of size)?
a. Almost none
b. A minority
c. About half
d. A majority
e. Almost all

\section{[Section 5-Other preferences]}


We will now ask you a series of questions about other preferences you have regarding your college/university's operations in Fall 2020.

5.1 Suppose that your college/university decided to shift the start and end dates of in-person course delivery for its Fall 2020 semester. Which option would be most preferable?

a. Begin classes in-person at the regular starting date and transition to online courses for the 3 weeks after the Thanksgiving break.

b. Begin classes online at the regular starting date and transition to in-person classes after 3 weeks. Classes resume in-person after the Thanksgiving break.

c. Begin classes in-person 3 weeks earlier than the regular starting date and finish the semester early at the Thanksgiving break (no planned transition to online courses).

5.2 Suppose that there was a $\mathbf{5 0 \%}$ chance that there would be an outbreak during the Fall $\mathbf{2 0 2 0}$ semester that required classes to be moved online for the remainder of the semester. In this situation, which of the options below do you prefer?

a. Start the semester with on-campus classes and move online if needed.

b. Deliver all courses online for the Fall 2020 semester

5.3 Suppose that there was a $\mathbf{7 5 \%}$ chance that there would be an outbreak during the Fall 2020 semester that required classes to be moved online for the remainder of the semester. In this situation, which of the options below do you prefer?

a. Start the semester with on-campus classes and move online if needed.

b. Deliver all courses online for the Fall 2020 semester

5.4 Suppose that there was a $\mathbf{1 0 0 \%}$ chance that there would be an outbreak during the Fall 2020 semester that required classes to be moved online for the remainder of the semester. In this situation, which of the options below do you prefer?

a. Start the semester with on-campus classes and move online if needed.

b. Deliver all courses online for the Fall 2020 semester 
5.5 If you were given a choice between two sections of the same lecture-based course (no lab component), one taught exclusively online from the beginning and the other taught in person when the semester starts, which one would you choose?

a. Exclusively online

b. In-person

5.6 If you were given a choice between two sections of the same lab-based course, one taught exclusively online using a simulated lab environment from the beginning of the semester and the other taught in-person when the semester starts, which one would you choose?

a. Exclusively online

b. In-person

5.7 Suppose that your college/university's campus was open, but due to the availability of online course options, you had a schedule that did not require your presence on-campus. Which of the following housing options would you choose?

a. Choose to live in on-campus housing

b. Choose to live off-campus but within commuting distance of campus

c. Choose to live outside commuting distance from campus

d. Defer enrollment

e. Other:

\section{[Section 6 - Spring 2020 Experience]}

6.1 Were you enrolled at a college/university in Spring 2020?

a. Yes

b. No

6.2 What was your living situation before your college/university closed in Spring 2020?

a. On-campus housing 
b. Off-campus housing, living independently of family

c. Off-campus housing, living with family

d. Other:

6.3 What was your living situation after your college/university closed in Spring 2020?
a. On-campus housing
b. Off-campus housing
c. Living remotely (e.g., living at home)
d. Other:

6.4 Where were you living after your college/university closed in Spring 2020?
a. within commuting distance;
b. outside commuting distance but within state;
c. out-of-state and not within commuting distance;
d. outside the US

6.5 How satisfied were you with online course delivery during the Spring 2020 term after your college/university moved courses online?
a. Extremely unsatisfied
b. Somewhat unsatisfied
c. Neither satisfied nor dissatisfied
d. Somewhat satisfied
e. Extremely satisfied

6.6 How would you rate your current living situation in terms of its ability to learn via remote (online) classes in Fall 2020 ? [Internet access, quiet space available, etc.] 

a. Excellent
b. Good
c. Average
d. Poor
e. Terrible

6.7 Where are you planning to live if courses resume on-campus in Fall 2020?
a. On-campus housing,
b. Off-campus housing, independently of family
c. Off-campus housing, living with family
d. Other:

\section{[Section 7: Perceived Risk and Comfort]}

7.1 What is the minimum level of social distancing in classrooms would you feel comfortable with if masks were required?
a. I would be comfortable without social distancing
b. 3 feet of distance
c. 6 feet of distance
d. 9 feet of distance
e. More than 9 feet of distance

7.2 What is the minimum level of social distancing in classrooms would you feel comfortable with if masks were not required?
a. I would be comfortable without social distancing
b. 3 feet of distance
c. 6 feet of distance
d. 9 feet of distance
e. More than 9 feet of distance 
7.3 How do you perceive your risk of being infected with COVID-19 during the Fall 2020 term if classes were held on campus?
a. Very low
b. Low
c. Moderate
d. High
e. Very high
f. I don't understand the question

7.4. How do you perceive your risk of having adverse effects from COVID-19 if you were to be infected?
a. Very low
b. Low
c. Moderate
d. High
e. Very high
f. I don't understand the question.

7.4 Do you have pre-existing conditions, asthma, or other conditions that put you at-risk for COVID-19 (coronavirus) complications?
a. Yes
b. No

\section{[Section 8 - Demographic Information]}

Your responses in this section enable us to expand the results from this small sample to the population as a whole. Your responses are anonymous, and we are not collecting any identifying information such as your IP address.

8.1 Are you...?

a. Female 
b. Male

c. Other (specify)

8.2 In what year were you born? (e.g., 1999)

8.3 How would you describe yourself? Please check ALL that apply to you.
a. Asian /Pacific Islander
b. Black / African American
c. Hispanic / Latino
d. Native American
e. White / Caucasian
f. Other (please specify)

8.4 Which best describes your academic standing in Fall 2020?
a. Freshman
b. Sophomore
c. Junior
d. Senior
e. Master's student
f. PhD student (Pre-candidate)
g. PhD student (Candidate)

8.5 Are you a Fall 2020 graduation candidate?
a. Yes
b. No 
8.6 What is your domestic/international student status?
a. Domestic student

b. International student

8.7 Are you receiving financial aid?
a. Yes
b. No

8.8. What are your parents' highest levels of education?
a. Some grade/high school
b. Completed high school or GED
c. Some college/technical school
d. Bachelor's degree
e. Some graduate school
f. Completed graduate degree(s)

8.9 How would you characterize the area where you grew up?
a. Urban part of a city/region
b. Suburban part of a city/region
c. Small town
d. Rural area

8.10 How would you characterize your current political leanings?
a. Democrat
b. Republican 
c. Independent

d. Other (please specify)

e. Decline to answer

8.11 Overall, how would you characterize your views?
a. Conservative
b. Moderate
c. Liberal
d. Decline to answer

\section{[Section 9 - Free Response]}

We would value any additional comments you may have on the Fall 2020 term at your college/university amid the COVID-19 pandemic or any other topics in this survey. Please write them in the space below.

\section{You have completed the survey. Thank you for your responses.}

